\title{
Predicting Interval Time for Reciprocal Link Creation using Survival Analysis
}

\author{
Vachik S. Dave • Mohammad Al Hasan . \\ Baichuan Zhang · Chandan K. Reddy
}

Received: date / Accepted: date

\begin{abstract}
The majority of directed social networks, such as, Twitter, Flickr, and Google+ exhibit reciprocal altruism, a social psychology phenomenon, which drives a vertex to create a reciprocal link with another vertex which has created a directed link towards the former. In existing works, scientists have already predicted the possibility of the creation of reciprocal link - a task known as "reciprocal link prediction". However, an equally important problem is determining the interval time between the creation of the first link (also called parasocial link) and its corresponding reciprocal link. No existing works have considered solving this problem, which is the focus of this paper. Predicting the reciprocal link interval time is a challenging problem for two reasons: First, there is a lack of effective features, since well-known link prediction features are designed for undirected networks and for the binary classification task, hence they do not work well for the interval time prediction; Second, the presence of ever-waiting links (i.e., parasocial links for which a reciprocal link is not formed within the observation period) makes the traditional supervised regression methods unsuitable for such data. In this paper, we propose a solution for the reciprocal link interval time prediction task. We map this problem to a survival analysis task and show through extensive experiments on real-
\end{abstract}

\author{
V. S. Dave, M. Al Hasan \\ Department of Computer \& Information Science \\ IUPUI, Indianapolis, USA \\ vsdave@iupui.edu, alhasan@cs.iupui.edu \\ Baichuan Zhang \\ Facebook \\ baichuan24@fb.com \\ C. K. Reddy \\ Department of Computer Science \\ Virginia Tech, Arlington, USA \\ reddy@cs.vt.edu
}


world datasets that survival analysis methods perform better than traditional regression, neural network based models, and support vector regression (SVR) for solving reciprocal interval time prediction.

Keywords Link Prediction · Directed Network · Reciprocity · Time Prediction · Survival Analysis

\section{Introduction}

Reciprocity is a phenomenon in social psychology which mandates that people should repay voluntarily what another person has provided for them. It is different from altruism (Anand et al, 2013) in the way that reciprocity follows from others' initial action, while altruism is a spontaneous action of gift-giving without the hope or expectation of future positive responses. There also exists another social psychology, named reciprocal altruism, which is a behavior whereby one performs an act of gift-giving with the expectation that the receiving person will act in a similar manner at a later time (Trivers, 1971). People's day-to-day activities on online social networks are filled with many examples of reciprocal altruism: we follow a friend's Twitter feed with the hope that he will follow back our feed; we like a friend's Facebook posts or her Flicker images with the expectation that she will do the same; we endorse our friends for their technical skill in LinkedIn hoping that they will return the favor in a similar manner.

However reciprocity usually is in conflict with another social phenomenon called social stratification, which favors hierarchical arrangement of people in a society based on various factors such as power, wealth, and reputation (Hopcroft et al, 2011). This phenomenon is prevalent in online social networks as well, but in a different manner. Apparently, for such networks, the social hierarchy is reflected in various prestige metrics which rank vertices based on their topological bearings, such as pagerank, and in-degree. Given this hierarchical arrangement in an online social network, people who are higher up in the hierarchy are sometimes reluctant to perform a reciprocal act for an individual who is lower in the hierarchy; they instead defer the reciprocal action to a later time, or sometimes indefinitely.

For reciprocal link creation, understanding the criteria which control the interval time and building learning models which predict the interval time are important. From a research standpoint, such studies help scientists to understand the interaction between reciprocity and social stratification phenomena. From the perspective of real-life applications in social network analysis, such prediction models enable better link suggestions, where the interval time is also factored in within the suggestion. Reciprocity, along with the interval time for reciprocal link creation, is particularly important for recommendation in online dating systems (Xia et al, 2015).

The majority of existing works on link prediction assume an undirected network (Hasan and Zaki, 2011; Valverde-Rebaza and de Andrade Lopes, 2013), in which the concept of reciprocal edges does not exist. A few works consider 
reciprocal link prediction (Hopcroft et al, 2011; Gong and $\mathrm{Xu}, 2014$ ) in a directed network where the prediction is binary, yielding a yes/no answer to the question of whether a reciprocal link will be created within a fixed observation window. Several other works utilize reciprocity as a tool for network compression (Chierichetti et al, 2009) and information propagation in social networks (Zhu et al, 2014). Reciprocal links also influence the degree correlations in complex networks, hence they play an important part in modeling the growth of directed social networks (Zlatić and Štefančić, 2009). However, none of the existing works consider predicting the interval time for the creation of a reciprocal edge.

Extending a model which solves a binary class reciprocal link prediction problem to a model which predicts the interval time of reciprocal links is non-trivial. The major challenge for interval time prediction is that typical link prediction features for an undirected network, such as common neighbors, Jaccard's similarity, and Adamic-Adar do not have a well-defined counterpart for directed networks, which makes interval prediction a difficult task. Additionally, for generating the training data for building a prediction model, a network is observed for a finite time window, and the absence of a reciprocal link within that time window does not necessarily mean the absence of that reciprocal edge, because a reciprocal edge might have formed outside (after) the observation time window. This yields numerous right censored data instances, for which the target variable, i.e., the reciprocal link formation time is not available. Traditional supervised regression models cannot include censored data instances in the training data and hence perform poorly in predicting reciprocal link creation time.

We explain the cases of right-censored data instances in reciprocal interval time prediction task using a toy example shown in Figure 1. In this figure we show a small part of an email communication network consisting of only three vertices representing three persons, $A, B$, and $C$. Our observation period of this network has five timestamps, $T 1$ to $T 5$. At $T 1, C$ sends an email to $B$, thus creating the first of the directed links (such links are called parasocial links). At $T_{2}$, the parasocial link from $A$ to $B$ is created. At $T 3$, the reciprocal link from $B$ to $C$ is created; thus the interval time of this edge is $T 3-T 1$. At $T 3$, another parasocial link $(B \rightarrow C)$ is created. More links are created in subsequent time intervals $T 4$ and $T 5$. At $T 5$, we reach the end of our observation period, but the reciprocal link from $C$ to $A$ is yet to be created. The potential reciprocal link $C \rightarrow A$ is an instance of right-censored data for which we only know that the interval time is higher than $T 5-T 1$; this value, as well, can be infinity in the case that the link is never created. Either way, the exact value of the target variable for this reciprocal edge is unknown. Unfortunately, for any reasonable observation time window, a significantly large number of potential reciprocal links are censored data instances, which is the main challenge for the task of reciprocal link creation time prediction.

In this work, we present a supervised learning model for predicting the interval time for the creation of a reciprocal edge between a pair of vertices in an online social network, given that a parasocial edge already exists between 


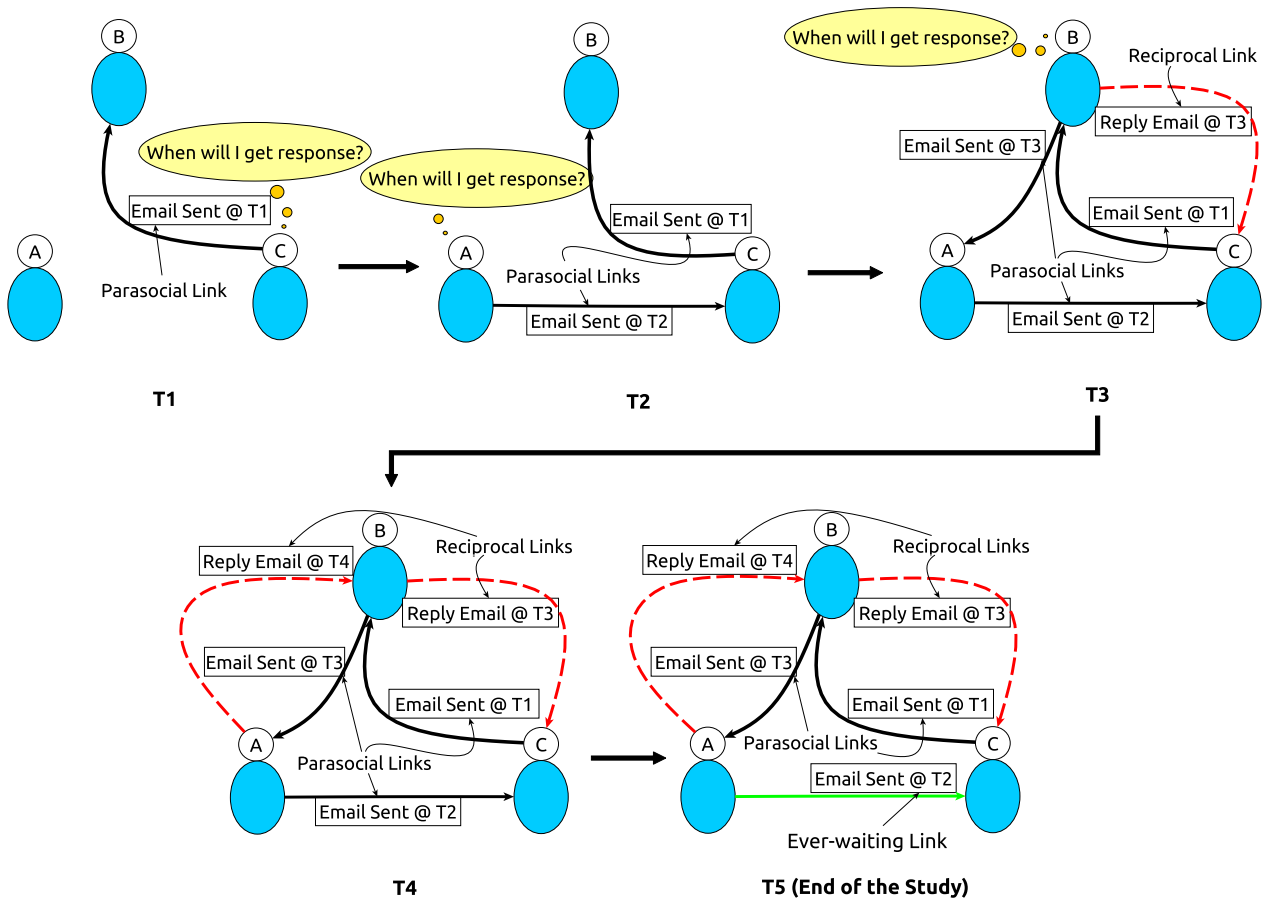

Fig. 1: An illustration of reciprocal link time prediction $R L T P$ problem.

the vertex-pair. We study real-life networks and validate a collection of topological features that may influence the reciprocal edge creation time. Then, we design the prediction task as a survival analysis problem and propose five censored regression models. Our experimental results show that Cox regression performs better than traditional supervised learning models for reciprocal link prediction. This is an extended version of our previous paper (Dave et al, 2017), which is published in 11th International Conference on Web and Social Media (ICWSM).

\section{Related Works}

The traditional binary classification task of link prediction has received enormous attention over the years since the inception of this problem by LibenNowell and Kleinberg in 2003 (Liben-Nowell and Kleinberg, 2003). Over the years researchers have solved the link prediction problem for a variety of graphs - for example link prediction in homogeneous networks (Hasan et al, 2006; Liaghat et al, 2013; Wang et al, 2017b), link prediction in heterogeneous information networks (Sun et al, 2011; Dong et al, 2012), and link prediction for knowledge graphs (Dong et al, 2014; Zhang et al, 2016). Other related problems, such as link/sign prediction and ranking in signed social network (Song 
and Meyer, 2015; Symeonidis and Mantas, 2013), and a recommendation system using link prediction techniques (Esslimani et al, 2011) have also been studied.

Reciprocal link prediction is a variant of link prediction which works on directed networks. Even though the majority of social and communication graphs are directed, only a few works exist which consider predicting reciprocal links. In one of the earliest works, J. Hopcroft et. al (Hopcroft et al, 2011) predicted reciprocal edges in a Twitter network. However, many of the features that they proposed are too specific to the Twitter dataset and do not apply to a generic directed network. N. Gong et. al (Gong and Xu, 2014) compared reciprocal and parasocial link creation in Google+ and Flickr datasets and solved the reciprocal link prediction problem as an outlier detection task using one-class SVM. Authors of (Cheng et al, 2011) compared structural differences of reciprocal links and parasocial links and they also studied a Twitter dataset and corresponding node features to predict reciprocal links. In another work (Feng et al, 2014), the authors reported that the majority of reciprocating links are created within a very short time after the creation of corresponding parasocial links. B. Dumba et al. (Dumba et al, 2016) studied the structural properties of a reciprocal network and discussed user behavior patterns.

A closely related problem to reciprocal link prediction is online dating recommendation. There exist a few works that solve this problem, mainly by using traditional recommendation methods with novel feature extraction processes. For example, in (Zhao et al, 2014) the authors modified the classical collaborative filtering method for the dating recommendation task. P. Xia et al. (Xia et al, 2015, 2016) proposed different reciprocal score matrices and used them with collaborative filtering for recommendation. The authors in ( Tu et al, 2014) proposed an LDA (Latent Dirichlet Allocation) based approach to learn latent preferences of users with two side matching based recommendation. Recently, X. Zang at al. (Zang et al, 2017) proposed a method that extracts profile-based features, topological features, and preference features from a dating social network for recommendation. All the existing works discussed so far target the binary classification problem, which predicts whether the reciprocal link will be created or not. On the other hand, our work targets the prediction of time, which is more difficult than the binary classification problem.

To the best of our knowledge, there are only two works that target the time prediction problem; the first one is by Y. Sun et al. (Sun et al, 2012) and the second by M. Li et al. (Li et al, 2016). In both of these works, the authors have extracted unique features for a DBLP-like (author-paper) heterogeneous network. Y. Sun et al. proposed meta path based topological features and used a generalized linear model (GLM) for the prediction task. Similarly, M. Li et al. proposed a novel time difference labeled path (TDLP) based method for the knowledge graph. Both methods are designed specifically for DBLP like networks, hence they are difficult to apply to other networks. On the other hand, our method is applicable to any general directed network to predict time of a reciprocal link. 


\section{Our Methodology}

In this section, we first define the problem of Reciprocal Link Time Prediction $(R L T P)$. Then we present some insight of three real world datasets that we have used in this work. Then we explain how the RLTP can be solved by using a survival analysis framework. After that we discuss different survival analysis methods which we have used for solving the RLTP problem. Finally, we provide algorithmic representation of the proposed framework.

\subsection{Problem Formulation}

Definition 1 Directed time-stamped network. Consider a network $G(V, E)$, where $V$ is the set of vertices and $E$ is the set of directed edges. $T$ is a set of time values, and $\tau$ is a mapping function, which maps an edge to one of the time values in the set $T$, i.e., $\tau: E \rightarrow T$. For an edge $e \in E, t_{e} \in T$ denotes the creation time of the edge $e$. Collectively, $G, T$, and $\tau$ are called a directed time-stamped network.

For vertices $u, v \in V$ and link $e=(u, v) \in E$ the corresponding time-stamp $t_{e}$ can be represented as $t_{u v}$. If an edge $e$ is created multiple times, we keep only the oldest (earliest) creation time and assign that to $t_{e}$. For a vertex $u \in V$, $\Gamma_{\text {in }}(u)$ and $\Gamma_{\text {out }}(u)$ are the set of in-neighbors and the set of out-neighbors of $u$, and $d(u, v)$ is the directed shortest path distance from $u$ to $v$.

Definition 2 Reciprocal/Parasocial Link. For a pair of vertices, $u$, and $v$, the edge $(u, v) \in E$ is called a parasocial link if the edge $(v, u) \notin E$. On the other hand, if $(v, u) \in E$ and $(u, v) \in E$, and $t_{v u}<t_{u v}$ then $(u, v)$ is called a reciprocal link.

The objective of the RLTP problem is to predict the time of a reciprocal link for the given parasocial link with time. The interval time for a reciprocal link $(u, v)$ is defined as $\operatorname{Int}(u, v)=t_{u v}-t_{v u}$. Our model for the RLTP problem actually predicts $\operatorname{Int}(u, v)$, instead of predicting $t_{u v}$ (the reciprocal link creation time). Nevertheless, the reciprocal link creation time $t_{u v}$ can be obtained from the model by using the expression $t_{v u}+\operatorname{Int}(u, v)$. The advantage of predicting $\operatorname{Int}(u, v)$ instead of predicting $t_{u v}$ is that for predicting $\operatorname{Int}(u, v)$ we do not need to use the parasocial link creation time $t_{v u}$ as part of input of the model, which makes the model independent of temporal bias. Thus the supervised model of our proposed RLTP task uses only the topological features of an edge $(u, v)$ as its covariates and the interval time $\operatorname{Int}(u, v)$ as its target variable, making the model simple.

\subsection{Dataset Study}

In this section, we discuss the datasets that we use in our study. We also provide some statistical analysis of the datasets; specifically, for each of these 
Table 1: Basic statistics of the datasets used in the paper.

\begin{tabular}{lcccc}
\hline Dataset & $|V|$ & $|E|$ & $|T|$ & Recipro \\
\hline Epinion & 131,828 & 841,373 & 938 & 0.3083 \\
MC-Email & 167 & 5,783 & 237 & 0.876 \\
Enron & 182 & 3,007 & 944 & 0.6053 \\
\hline
\end{tabular}

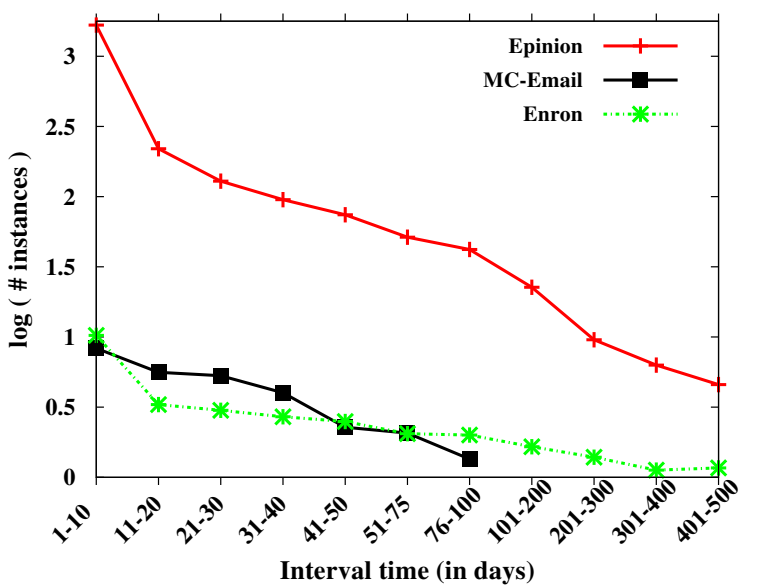

Fig. 2: Histogram of interval time of reciprocal link.

datasets, we provide the empirical distribution of observed interval time and its goodness of fit with known statistical distributions. For the Enron dataset, the persons (along with their rank in the company) associated to a vertex is known, so in this dataset we have also performed a qualitative study by checking for the evidences of social stratification phenomenon, which we present at the end of this section.

We used three real-world directed network datasets for our study. We selected datasets where reciprocal link creation is an important (meaningful) event; another selection criterion is that the datasets should have a sufficient number of reciprocal links to train and test the models (Kuhnt and Brust, 2014). Our first dataset, Epinion is a trust network where a directed link from one vertex to another represents the fact that the former trusts the latter. The RLTP task for this dataset is to find the time at which a trusted person acknowledges that (s)he also holds a similar sentiment towards the other person. The dataset was collected from KONECT web page. ${ }^{2}$ We have also collected two email datasets: $M C$-Email ${ }^{3}$ and Enron. Both of these datasets are email communication networks from two distinct enterprises, and for these datasets the RLTP task is to predict the response time for an email. More information on these datasets is provided in Table 1 , where $|V|,|E|,|T|$, and Recipro

\footnotetext{
2 http://konect.uni-koblenz.de/networks/

3 This is Manufacturing Company email dataset available from R. Michalski's website, https://www.ii.pwr.edu.pl/ michalski
} 
are the number of vertices, the number of edges, the number of timestamps (in days), and the reciprocity of the dataset within the observation window, respectively.

For these three datasets, we plot the histogram of the interval time for reciprocal links in log scale (Figure 2). We observed that the majority of the responses are received within a short period of time (within 10 days or less). However, there also exist a few late responders whose reply time is much larger than the average reply time.

\subsubsection{Modeling interval time using Parametric Distribution}

From the distribution plots in Figure 2 we observe that the number of reciprocal link instances reduces exponentially with the increment of the interval time (note that, y-axis is in log-scale). Hence, we fit different exponential family distributions to model the time interval of reciprocal link for all three datasets. Specifically, we fit exponential distribution, normal distribution, logistic distribution, log-normal distribution, log-logistic distribution and Weibull distribution. To evaluate the goodness of fit we use the follow-

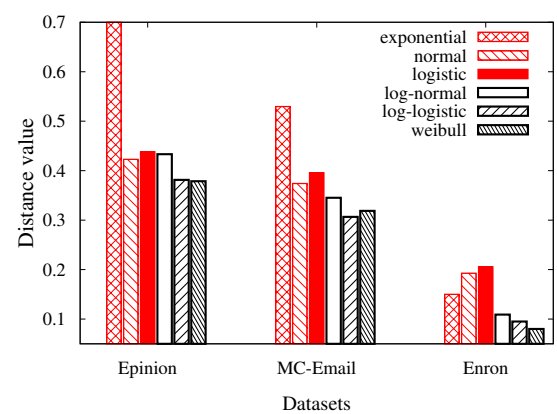

(a) Kolmogorov-Smirnov statistic

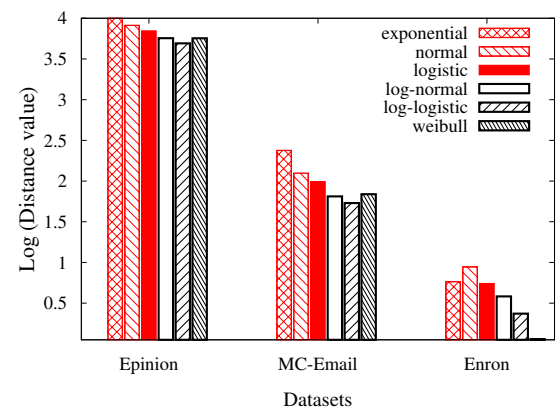

(b) Cramer-von Mises statistic

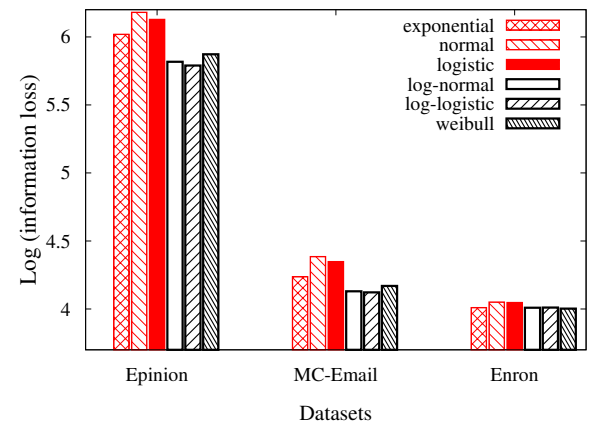

(c) Aikake's Information Criterion

Fig. 3: Goodness of fit comparisons for different distributions. 
ing four metrics: Kolmogorov-Smirnov (KS) statistic, Cramer-von Mises (CM) statistic, Aikake's Information Criterion (AIC) (Akaike, 1998) and Bayesian Information Criterion (BIC) (Schwarz, 1978). In Figure 3, we show the quality of fitting results. The results of BIC are very similar to AIC for all three datasets, so we did not show the results of BIC. As depicted in Figure 3, exponential, normal, and logistic distributions (shown in red) have relatively high distance from empirical distribution compared to log-normal, log-logistic and Weibull distributions (shown in black). For the Enron dataset, Weibull distribution performs the best over all metrics. Similarly, for the Epinion and the $M C$-Email datasets log-logistic distribution fits the best. Results of lognormal distribution are very similar to both Weibull and log-logistic distributions. Hence, we use log-normal, log-logistic and Weibull distributions for parametric survival models, which are discussed later in Section 3.5.

\subsubsection{Social Stratification in Enron}

One of the influencing factors for late responses to a specific user is social stratification - particularly in corporations, people tend to give quicker replies to their superior as compared to their colleagues and other juniors. We study the Enron dataset, for which the employee details are available with email communications. In the dataset, "Louise Kitchen" is a president; we observed that her email replying practice follows social stratification phenomenon. She generally takes more than $2-3$ days to reply to people with lower ranking positions such as vice-president (VP), employees, etc. For example, she replied to VPs "Kevin Presto", "James Steffes" and "Fletcher Sturm" in 3, 6 and 19 days respectively. She replied to "Sally Beck" (Chief Operating Officer) in 5 days. On the other hand she replied to "David Delainey" (Chief Executive Officer (CEO)) on the same (0) day. Another example is "Philip Allen", who is a manager; he replied within a day to higher ranking officers such as "David Delainey" (CEO), "Barry Tycholiz" (VP), "Hunter Shively" (VP) and "Richard Shapiro" (VP). On the other hand, he took 2 to 3 days to reply to "Michael Grigsby" (manager), "Jay Reitmeyer" (employee) and "Matthew Lenhart" (employee).

\subsection{Topological Feature Study}

In online social networks, user behavior based features are useful for solving different problems, such as, link prediction (Valverde-Rebaza and de Andrade Lopes, 2013), personality prediction (Adalı and Golbeck, 2014), user attribute prediction (Tuna et al, 2016), link sign prediction (Shahriari et al, 2016), prediction of positive and negative users in Twitter (Roshanaei and Mishra, 2015), etc. Hence, we believe social (behavioral) phenomena based topological features can contribute substantially to solve the RLTP problem. Though there are works that study and design user behavior features such as 
topic-specific modeling (Bogdanov et al, 2014), a behavioral model for Facebook wall posts (Devineni et al, 2017), etc., we assume to have only topological information. Topological features that we use comes from two different social phenomena: directed altruism and social stratification. Below we discuss them in two different sections.

\subsubsection{Directed Altruism Based Features}

Directed altruism in social networks is described in (Leider et al, 2007), where the authors have argued that people are more generous to friends and friends of friends than to a complete stranger. This phenomenon also reflects in people's reciprocal link creation behavior. Below, we define some topological features which quantify the directed altruism phenomena for reciprocal link prediction.

Shortest directed distance: In our problem, one directional link $(v, u)$ already exists, and we are predicting the creation time for the reverse link $(u, v)$. Generally people are more generous to indirect friends than complete strangers. Hence $u$ is more likely to respond quickly to $v$ for small value of the directed distance from $u$ to $v$ i.e.

$$
\text { DirectedDist }(u, v)=d(u, v)
$$

Common in/out neighbors count: The number of common neighbors is a frequently used topological feature for the link prediction task in undirected networks; however, for directed graphs, we have two separate features: common in-neighbors and common out-neighbors. Both of these topological features capture the idea that if a user has more common neighbors with another user, then she is more likely to reply fast. Also, more common friends increase the network flow, which is an important factor for building trust (Leider et al, 2007) and with higher trust people tend to reply faster.

$$
\begin{gathered}
\operatorname{Common}_{\text {in }}(u, v)=\left|\Gamma_{\text {in }}(u) \cap \Gamma_{\text {in }}(v)\right| \\
\text { Common }_{\text {out }}(u, v)=\left|\Gamma_{\text {out }}(u) \cap \Gamma_{\text {out }}(v)\right|
\end{gathered}
$$

Jaccard coefficient (In/Out): The Jaccard coefficient is another widely used topological feature for undirected networks. It is the normalized version of common neighbors counts. Similar to the common neighbor count feature, this feature also split into two features due to the directed-ness of the edges. Jaccard coefficients help to predict the trust level between two nodes. Since, higher trust leads to faster response, this is a good feature for the RLTP task.

$$
\begin{aligned}
\operatorname{Jaccard}_{\text {in }} & =\frac{\left|\Gamma_{\text {in }}(u) \cap \Gamma_{\text {in }}(v)\right|}{\left|\Gamma_{\text {in }}(u) \cup \Gamma_{\text {in }}(v)\right|} \\
\operatorname{Jaccard}_{\text {out }} & =\frac{\left|\Gamma_{\text {out }}(u) \cap \Gamma_{\text {out }}(v)\right|}{\left|\Gamma_{\text {out }}(u) \cup \Gamma_{\text {out }}(v)\right|}
\end{aligned}
$$

Local Reciprocity. In (Gong and $\mathrm{Xu}, 2014$ ), the authors studied two local reciprocity features and they showed relative influence of both features 
on linking back probability. The first is Acceptance Local Reciprocity $(A L R)$, which is defined as:

$$
A L R(v)=\frac{\left|\Gamma_{\text {in }}(v) \cap \Gamma_{\text {out }}(v)\right|}{\left|\Gamma_{\text {in }}(v)\right|}
$$

We compute $A L R$ for the head node $(v)$ of the reciprocal link $(u, v)$. This feature captures the tendency of node $v$ to accept a link. The second feature is Request Local Reciprocity $(R L R)$, defined as:

$$
R L R(u)=\frac{\left|\Gamma_{\text {in }}(u) \cap \Gamma_{\text {out }}(u)\right|}{\left|\Gamma_{\text {out }}(u)\right|}
$$

We compute $R L R$ for the tail node $(u)$ of the reciprocating link $(u, v) . R L R$ represents the response behavior of the node $u$ and captures its tendency to initiate a reciprocal link.

\subsubsection{Social Stratification Based Features}

It is observed that in online social networks people behave according to their status in the network (Hopcroft et al, 2011). A similar behavior is observed in many real world applications, such as the one described in Section 3.2 or in online dating (Xia et al, 2013). We have also shown evidence of social stratification in Enron dataset, specifically in connection to the RLTP task. The following topological features quantify the extent of social stratification that is practiced by the node $u$ or $v$.

Preferential Attachment: This feature computes a value which reflects the social stratification induced rank order of a given node. The basic idea of preferential attachment is to give more weight to the higher degree nodes. Traditionally, preferential attachment has been computed for undirected networks, so we change the formula to adapt it for a directed networks. For undirected graph, it is simply the product of the degrees of the node $u$ and $v$. For directed graph, we take the product of the out-degree of the tail node $(u)$ and the in-degree of the head node $(v)$ of a prospective reciprocal link $(u, v)$. The formula is given below:

$$
\text { Pref } \operatorname{Att}(u, v)=\left|\Gamma_{\text {out }}(u)\right| \times\left|\Gamma_{\text {in }}(v)\right|
$$

Preferential Jaccard: PrefJacc is inspired by both Preferential Attachment and Jaccard Coefficient. It is a trade-off between two concepts - first, high degree nodes are prone to create more edges, and second, nodes prefer to connect with similar nodes (social stratification). Both these phenomena can influence reciprocal edge creation. We calculated PrefJacc by using the following equation:

$$
\operatorname{Pref} \operatorname{Jacc}(u, v)=\frac{\left|\Gamma_{\text {out }}(u) \cap \Gamma_{\text {in }}(v)\right|}{\left|\Gamma_{\text {out }}(u) \cup \Gamma_{\text {in }}(v)\right|}
$$

In/Out Ratio: A node in the upper hierarchy has a tendency to a create reciprocal edges with other nodes at the same hierarchy level than to nodes 
which are at a lower hierarchy level (Hopcroft et al, 2011). To reflect this knowledge in our model, we need to find an efficient way for comparing the hierarchy of a pair of nodes, which we compute by the ratio of their in-degrees and the ratio of their out-degrees. Higher InRatio is indicative of higher tendency of the numerator node to attract links compared to the denominator node; similarly, higher OutRatio represents a higher tendency of the numerator node to create links compared to the denominator node. In this way, these two features capture the relative patterns of link creation and link acceptance by the pair of the vertices. For reciprocating link $(u, v)$, we calculate InRatio and OutRatio by using the following equations:

$$
\begin{gathered}
\text { InRatio }=\frac{\left|\Gamma_{\text {in }}(u)\right|}{\left|\Gamma_{\text {in }}(v)\right|} \\
\text { OutRatio }=\frac{\left|\Gamma_{\text {out }}(u)\right|}{\left|\Gamma_{\text {out }}(v)\right|}
\end{gathered}
$$

PageRank: PageRank represents the prestige of the node in the network. We use both, pagerank of $u$ and pagerank of $v$ as features. If PageRank(u) is lower than PageRank(v), then the node $u$ is highly likely to respond faster to the node $v$.

\subsubsection{Feature analysis}

To validate the strength of these features (13 in total) for predicting the interval time of reciprocal edges, we compute the Pearson's correlation of the above topological features with the interval time value for three real-life graph datasets (Table 1) and show the correlation values in Table 2. As we can see, for the $M C$-Emails dataset most of the features (mainly Common ${ }_{\text {in }}, C_{\text {Common }}$ out , Jaccard $_{I n}$, Jaccard $_{\text {Out }}$, PrefAtt and PrefJacc) have good correlation value (between 0.2 to 0.5). Similarly, for the Enron dataset the same set of features

Table 2: Correlation of features with Interval time

\begin{tabular}{l|c|c|c}
\hline Features/ Datasets & Epinion & MC-Emails & Enron \\
\hline DirectedDist $_{\text {Common }}$ In & -0.04127 & -0.03792 & -0.13336 \\
Common $_{\text {Out }}$ & 0.38109 & 0.33447 & 0.44398 \\
Jaccard $_{\text {In }}$ & 0.27254 & 0.31194 & 0.27534 \\
Jaccard $_{\text {Out }}$ & 0.17161 & 0.22101 & 0.24831 \\
RLR(u) & 0.11015 & 0.18925 & 0.20195 \\
ALR $(v)$ & -0.00290 & 0.05820 & 0.16053 \\
PrefAtt $_{\text {PrefJacc }}^{-0.06093}$ & 0.15383 & 0.19256 \\
InRatio $_{\text {OutRatio }}$ & 0.19289 & 0.23930 & 0.25443 \\
PageRank(u) $_{\text {PageRank(v) }}$ & -0.09136 & 0.20054 & 0.25502 \\
& -0.01132 & -0.07053 & -0.14302 \\
& 0.24783 & 0.04269 & 0.13108 \\
& 0.14300 & 0.07523 & -0.07609 \\
& \multicolumn{3}{|c}{}
\end{tabular}



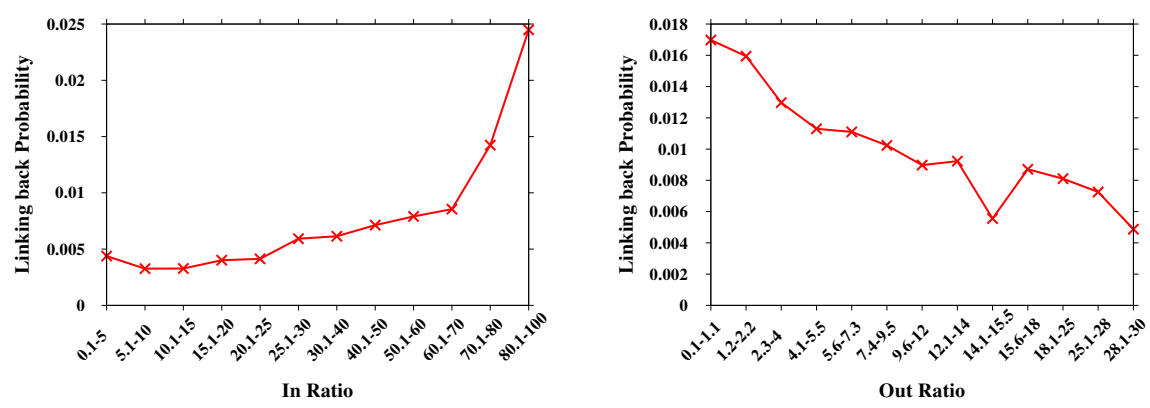

Fig. 4: Relation of In/OutRatio and linking back probability in Epinion dataset

is highly related to interval time. But, for Epinion dataset the correlation values for most of the features are poor except for Common ${ }_{i n}, C_{o m m o n}$ out , and PageRank(u); the worst features are InRatio, OutRatio and RLR(u). To check the influence of these features on reciprocal link creation, we also check the average linking back probability over different range of values for different features. We discuss our observation in the following paragraphs.

In Figure 4, we plot our observation for two of the features: InRatio and OutRatio. Here, for each bin of InRatio, the linking back probability is calculated as a fraction of reciprocal links over all the links in that bin. Figure 4 clearly shows high linking back probability for higher InRatio and lower OutRatio, which is expected behavior for these features. In (Gong and $\mathrm{Xu}, 2014$ ), the authors provided a thorough study of some features, such as, $R L R(u)$ and $A L R(v)$, and proved their significant influence on reciprocal link creation.

in Figure 5, we show three plots (one for each dataset) of DirectedDist vs. interval time. Within each plot we have several graphs, each representing the directed distance value between the vertices. Along the $\mathrm{x}$-axis is the interval time and along the $\mathrm{y}$-axis is the number of reciprocal link instances that have the corresponding interval time. For all dataset, we observe that links with small directed distance value (such as, 2 or 3 ) can have high interval time, i.e. the reciprocal link may appear after many days; but as distance increases there are few or almost no instances of reciprocal links with high interval time. This observation may appear counterintuitive as we expect short distance to influence a short interval time. However, This observation can be explained as follows: people tend to trust other people who are within their circles, and they will ultimately create a reciprocal links with them, even if they do not do it immediately. On the other hand, for people who are outside someone's circle (having a high directed distance value, such as 4 or 5), reciprocal links will be created either in a short interval time or will not be created at all. The short interval time can be the cases when two strangers meet in-person in a social event and then mutually agree to be connected online (or trust each other). On the other hand, the negative case happens, when a stranger trusts (or sends an invite to) someone, and the second person just ignore that forever. Due to 


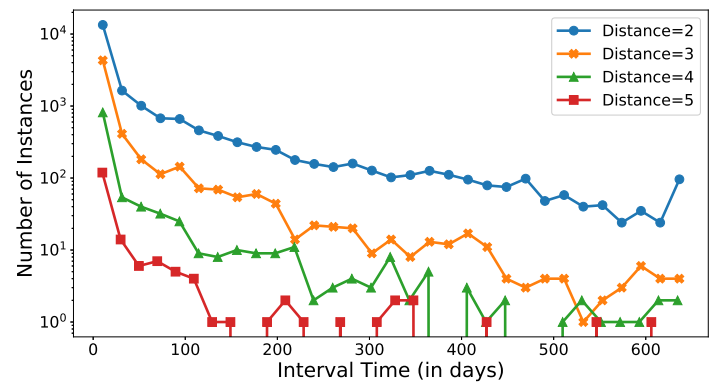

(a) Epinion Dataset

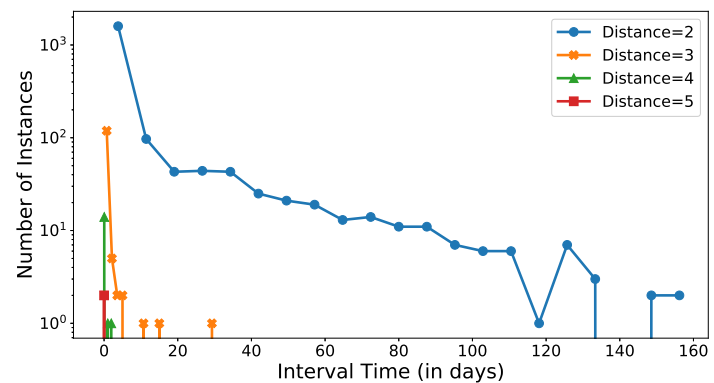

(b) $M C$-Email Dataset

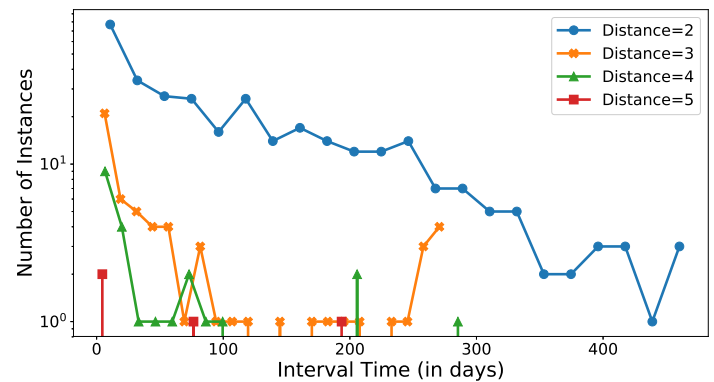

(c) Enron Dataset

Fig. 5: DirectedDist vs. Interval Time

this complex relation, the correlation between directed distance and interval time is poor, yet we consider DirectedDist to be a useful feature.

\section{Correlation with Low and High Interval time.}

There are a variety of different social behaviors that influence the interval time, hence some social based features impact the interval time differently over a period. To understand the impact of different features over a period, we split the target variable (interval time) into lower and higher range and 
Table 3: Correlation of features with Low and High Interval times

\begin{tabular}{|c|c|c|c|c|c|c|}
\hline \multirow{2}{*}{$\begin{array}{l}\text { Datasets } \\
\text { Features }\end{array}$} & \multicolumn{2}{|c|}{ Epinion } & \multicolumn{2}{|c|}{$M C$-Emails } & \multicolumn{2}{|c|}{ Enron } \\
\hline & Low & High & Low & High & Low & High \\
\hline DirectedDist & -0.00387 & -0.04587 & 0.14453 & -0.06022 & -0.04023 & -0.15364 \\
\hline Common $_{\text {In }}$ & 0.06671 & 0.33821 & 0.00018 & 0.41728 & 0.22168 & 0.35640 \\
\hline Common $_{\text {Out }}$ & 0.07231 & 0.24064 & 0.06639 & 0.27446 & 0.04738 & 0.20793 \\
\hline Jaccard $_{I n}$ & 0.07765 & 0.15312 & -0.04154 & 0.29774 & 0.17726 & 0.10033 \\
\hline Jaccard $_{\text {Out }}$ & 0.06829 & 0.13183 & -0.07426 & 0.22517 & 0.07820 & 0.06360 \\
\hline$R L R(u)$ & -0.03937 & 0.06628 & -0.17897 & 0.02467 & 0.07949 & 0.06348 \\
\hline$A L R(v)$ & -0.01783 & -0.07657 & 0.15905 & 0.09455 & 0.08760 & 0.06401 \\
\hline PrefAtt & 0.03049 & 0.14439 & -0.00163 & 0.31220 & 0.06305 & 0.32053 \\
\hline PrefJacc & 0.04258 & 0.13021 & -0.06545 & 0.23248 & 0.16523 & 0.09010 \\
\hline InRatio & -0.01251 & -0.01751 & -0.15333 & -0.04297 & -0.10385 & -0.09571 \\
\hline OutRatio & -0.00700 & -0.02610 & 0.29600 & -0.06979 & 0.00578 & 0.12331 \\
\hline PageRank(u) & 0.06118 & 0.20674 & -0.07606 & -0.09756 & -0.00557 & -0.12452 \\
\hline PageRank(v) & 0.02399 & 0.14362 & 0.31830 & -0.13432 & 0.01606 & 0.03715 \\
\hline
\end{tabular}

calculate feature correlations with lower and higher interval times separately. For this study, we calculate average interval time for each dataset and if the interval time is less or equal to average interval time we call it low interval time, otherwise, we call it high interval time. For each dataset and each feature we calculate the correlation value between the feature and low and high interval times; these correlation values are shown in Table 3.

In Table 3, we observe that features like Common in, Common $_{\text {out }}$, Jaccard $_{\text {In }}$, Jaccard $_{\text {Out }}$, PrefAtt and PrefJacc have high correlation with higher interval time. For the Enron dataset, some of these features $\left(\right.$ Common $_{\text {in }}$, Jaccard $_{I n}$ and PrefJacc) are highly correlated to lower interval time as well. For the $M C$-Email dataset, DirectedDist, ALR(v), Out Ratio and PageRank(v) have noticeable correlation with lower interval time and other two features $(R L R(u)$ and In Ratio) are inversely correlated to lower interval time. One surprising observation for the $M C$-Email dataset is that PageRank(v) is the poorest feature (Table 2), but highly correlated with both lower and higher interval times, mainly because the feature is positively correlated for lower interval time and inversely correlated with higher interval time. From Table 3 we understand that for different datasets user behavior varies and hence a distinct set of features becomes influential to the interval time (especially lower interval time) of that dataset.

\subsection{Proposed methodology using survival analysis}

Survival analysis is widely used in the medical domain to predict survival time or time to a specific event (such as death) for patient datasets (Vinzamuri and Reddy, 2013), (Wang et al, 2017a). In the survival analysis setup, for a set of instances under observation, events happen over a time period, from which a survival model learns the temporal patterns of these events and predicts the survival time. Here, we propose a novel method to map the RLTP problem 
to a survival analysis task and explain survival analysis concepts from a reciprocal link creation perspective. For these concepts, we also provide suitable terminology for the RLTP problem to describe our approach clearly.

Beginning of graph expansion and study period: At the first timestamp, a given directed time-stamped network is static (initialized); the beginning of graph expansion is the second time-stamp from when new links are added to the static network. Survival analysis assumes a starting time of the study, from when a model starts to observe for the events. In the RLTP problem, the beginning of graph expansion serves as the starting time of the study. For the RLTP problem, we divide the time-stamps of the network into train and test time periods, and we observe the network for the reciprocal link creation till the end of the train period, so the last time-stamp in the train period is considered to be the end of the study. Thus the time window from the beginning of graph expansion to the last time-stamp of train period is considered to be the study period which is the same as the train period.

Reciprocal event: For a parasocial link $(v, u)$, if a reciprocal link $(u, v)$ is created during the training period, we call it a reciprocal event, which is the event of interest in the RLTP problem. In the RLTP problem each parasocial link is a data instance, time-stamp of a parasocial link generation is the time when the data instance is considered into the network for study. Hence, the time-stamp of a parasocial link generation is called the starting time of observation for that data instance (an ordered pair of vertices).

ever-waiting links: We study the network for a limited time window (train period), and hence for a set of parasocial links, the corresponding reciprocal event may not be observed before the end of the study (last time-stamp of training period). We call these links ever-waiting links. ever-waiting links carry the information that the reciprocal link creation event did not happen till the end of the train period. In the survival analysis terminology the everwaiting links are also called censored instances; we use both of these terms interchangeably in this paper.

In a traditional regression task, ever-waiting links may either be ignored, because the target value (the interval time) for these instances are unknown, or they may be retained with an arbitrarily chosen large interval time, which is higher than the time difference between the end of the study time and the starting time of observation for that parasocial link. The first of the above approaches ignores important information; specifically, the ignored fact is that the interval time for ever-waiting links is higher than the time difference between the end of the study and the starting time of observation for that parasocial link. The second approach is simply a crude approximation of the target value. As mentioned before, the main reason to map the RLTP problem into survival regression analysis framework is to exploit the important information provided by the ever-waiting links. 
Target value of survival regression model: The time difference between the starting time of observation (parasocial link generation time) and the timestamp of the reciprocal edge creation is the interval time which we want to predict in the RLTP task. For a reciprocal edge $(u, v)$, the interval time is defined as $\operatorname{Int}(u, v)$, as is discussed in Section 3.1. In a traditional survival model, the interval time is called the life-span of an instance as for these models "death" is the event of interest. Hence survival models that predict survival time can be adopted to predict the interval time for the RLTP problem. For training the prediction model, we need a feature vector for each data instance, along with the survival time and a binary event indication value (event occurred or not). For the RLTP problem, the feature vector of a parasocial edge is $\mathbf{x}_{i} \in \mathbb{R}^{d}$, a vector of topological features (Section 3.3) for the $i$ 'th parasocial link in training data, where feature dimension $d$ is 13 (number of topological features). For each parasocial links of the training period, if the reciprocal event has occurred during training period then life-span of parasocial link is the interval time with the event indication value set to 1 ; otherwise, for everwaiting links, the time difference between the last time-stamp of training and time-stamp of the parasocial link generation is the survival time with event indication value set to 0 . Given this training dataset the target value (the interval time) of test instances are predicted by using a trained survival model. We use various survival models, which we discuss in the next subsection.

\subsection{Survival models for the RLTP problem}

As explained in the previous section, any survival model can be adopted to solve the RLTP problem. There are two types of widely used survival models: 1) semi-parametric models and 2) parametric models. Parametric models assume that interval time follows a known statistical distribution; hence, if the interval time for a dataset follows a distribution then parametric models perform very good for the dataset compared to a semi-parametric models. However for many real-world datasets, it is difficult to find a suitable statistical distribution that fits well to the interval time, for these datasets semi-parametric models perform better than parametric models, because semi-parametric models do not assume any underlying distribution, rather they try to learn the actual distribution from the data. As we discussed in Section 3.2, some of our datasets are good fit for a statistical distribution but others are not. Hence, we conduct experiments with both semi-parametric and parametric models to offer a comprehensive study of the RLTP problem. In this section, we describe these selected semi-parametric and parametric models and their adaptation for solving the RLTP problem. Broadly, all types of models try to predict the survival time of an instance in the data by modeling three functions: 1) Survival function, 2) Hazard function and 3) Event density function. Definitions and relationship between these three functions are described below: 
Survival function $S(t)$ Survival models provides a principled approach for interval time prediction by modeling a survival function, which is defined as the probability value that the reciprocal edge creation does not happen for a given parasocial link before a specified time $t$.

$$
S(t)=\operatorname{Pr}(\boldsymbol{T} \geq t)
$$

Here, $\boldsymbol{T}$ is a random variable representing the time of the reciprocal edge creation event.

Hazard function $\lambda(t)$ is the reciprocal event rate at time $t$ conditional on the fact that the reciprocal event has not occurred until that time $t$,

$$
\lambda(t)=\frac{f(t)}{S(t)}
$$

where $f(t)$ is the reciprocal event density function, which is given as follows:

$$
f(t)=\frac{d}{d t}(1-S(t))=-\frac{d}{d t} S(t)
$$

For a given parasocial link if corresponding reciprocal link is not likely to be created at time $t$ then Survival function value for $t$ is high. On the other hand, if the corresponding reciprocal link is highly likely to be created at time $t$ then the reciprocal event density function value should be high and that leads to a higher value of the Hazard function. We can observe that both survival function and hazard function are interrelated and we can model either function for the interval time prediction. Next, we describe how semi-parametric Cox regression models the hazard function to solve the RLTP problem. Later we discuss parametric methods (BJ-model and AFT models) and their approach for modeling the survival function with the help of different statistical distributions.

\subsubsection{Cox Regression}

Cox regression model (Cox, 1972) is the most widely used semi-parametric model for predicting the (interval) time taken for a reciprocal event to occur. The basic Cox model follows the proportional hazard assumption, for which the hazard function $\lambda\left(t \mid \mathbf{x}_{i}\right)$ takes the following form:

$$
\begin{aligned}
\lambda\left(t \mid \mathbf{x}_{i}\right) & =\lambda_{0}(t) \times \exp \left(\beta_{1} x_{i 1}+\beta_{2} x_{i 2}+\ldots+\beta_{d} x_{i d}\right) \\
& =\lambda_{0}(t) \times \exp \left(\mathbf{x}_{i}^{T} \boldsymbol{\beta}\right)
\end{aligned}
$$

where, $\mathbf{x}_{i}$ is the (topological) feature vector of a parasocial link represented as $i$ 'th data instance in the training data and $d$ is the dimensionality of the features. Here, $\lambda_{0}(t)$ is called baseline hazard function, and $\boldsymbol{\beta}$ is the model parameter which Cox regression model learns. The Cox regression is called semi-parametric because the baseline hazard function $\lambda_{0}(t)$ can be any nonnegative function of time. The probability of occurrence of reciprocal event for 
the $i^{\text {th }}$ parasocial link (data instance) at time $t$ can be represented as ratio $\frac{\lambda\left(t \mid \mathbf{x}_{i}\right)}{\sum_{j \in R_{t}} \lambda\left(t \mid \mathbf{x}_{j}\right)}$, where $R_{t}$ is the set of all instances for which the reciprocal event did not happen until $t$. The product of these probabilities gives the partial likelihood function:

$$
L(\boldsymbol{\beta})=\prod_{i=1}^{N}\left[\frac{\exp \left(\mathbf{x}_{i}^{T} \boldsymbol{\beta}\right)}{\sum_{j \in R_{t}} \exp \left(\mathbf{x}_{j}^{T} \boldsymbol{\beta}\right)}\right]^{C_{i}}
$$

Here, $N$ is the total number of parasocial links appeared during the training period and $C_{i}$ is an event indicator value, i.e., if reciprocal link for the $i^{\text {th }}$ parasocial link appear during training period then $C_{i}=1$ otherwise $C_{i}=0$. The model parameter $\boldsymbol{\beta}$ is learnt by minimizing the negative log likelihood function. If $\widehat{\boldsymbol{\beta}}$ is the optimal model parameter, we have:

$$
\widehat{\boldsymbol{\beta}}=\underset{\boldsymbol{\beta}}{\operatorname{argmin}} \frac{1}{N} \sum_{i=1}^{N}\left[-C_{i}\left(\mathbf{x}_{i}^{T} \boldsymbol{\beta}\right)+C_{i} \log \left(\sum_{j \in R_{t}} \exp \left(\mathbf{x}_{j}^{T} \boldsymbol{\beta}\right)\right)\right]
$$

Regularized Cox model: For complex model with high dimensional real world datasets, over-fitting is a frequent problem. To avoid this, we need a regularization term in the objective function (Equation 4). We observe in Section 3.3.3 that only a few features have a strong correlation with the target variable, so we want to use a sparse regularization model. In this work we use Elastic Net regularization. In literature, a Cox model with Elastic Net regularization is also known as Cox model with Elastic Net (EN) penalty (Zou and Hastie, 2005). The penalty term $P_{E N}$ is:

$$
P_{E N}(\boldsymbol{\beta})=\sum_{k=1}^{d}\left[\alpha\left|\beta_{k}\right|+\frac{1}{2}(1-\alpha) \beta_{k}^{2}\right]
$$

Where, $0<\alpha \leq 1$ and with EN penalty the objective function in Equation 4, becomes

$$
\widehat{\boldsymbol{\beta}}=\underset{\boldsymbol{\beta}}{\operatorname{argmin}} \frac{1}{N} \sum_{i=1}^{N}\left[-C_{i}\left(\mathbf{x}_{i}^{T} \boldsymbol{\beta}\right)+C_{i} \log \left(\sum_{j \in R_{t}} \exp \left(\mathbf{x}_{j}^{T} \boldsymbol{\beta}\right)\right)\right]+\gamma \cdot P_{E N}(\boldsymbol{\beta})
$$

Here, $\gamma>0$ is a regularization constant. For solving this optimization task, we can use the maximum partial likelihood estimator proposed in (Cox, 1972); it uses the Newton-Raphson method to iteratively find the estimated $\widehat{\boldsymbol{\beta}}$ which minimizes the Equation (6). 


\subsubsection{Parametric Models}

The main idea behind a parametric model is that it assumes that the interval time follows a specific statistical distribution. There are two ways to relate interval time and a statistical distribution: first, assume that the actual interval time for all parasocial links follows a distribution; and second, assume that the logarithm of the interval time follows a distribution. The models under the first assumption are referred to as linear regression models, and the models under later assumption are called accelerated failure time (AFT) models.

Generally, parametric models use maximum likelihood estimation (MLE) approach to learn model parameters. Let's assume all the parameters of a model are represented by $\boldsymbol{\beta}=\left(\beta_{1}, \beta_{2}, \ldots\right)^{T}$. For a given parasocial link (say $i^{\text {th }}$ link in the training data), if it is an ever-waiting link the corresponding survival function $S(t, \boldsymbol{\beta})$ at time $t$ (in fact, any $t$ value during a training period) should be near to 1 , and if it is not an ever-waiting link then the reciprocal event density function $f\left(t_{i}, \boldsymbol{\beta}\right)$ at time $t_{i}$ (time of reciprocal event for the $i^{t h}$ parasocial link) should be high (near to 1 ) for that link. Hence, the likelihood of all parasocial links of a training period is the product of their reciprocal event density functions or survival functions based on their state (whether the link is ever-waiting or not), i.e.

$$
L(\boldsymbol{\beta})=\prod_{C_{i}=1} f\left(t_{i}, \boldsymbol{\beta}\right) \cdot \prod_{C_{i}=0} S\left(t_{i}, \boldsymbol{\beta}\right)
$$

Linear regression model: The statistical linear regression with the least squares estimation is widely used for a variety of regression tasks. However, the issue with the model is that it cannot use information from ever-waiting links. For interval time prediction this issue can be handled by using a specific survival model such as the Buckley-James model (BJ model). The BJ model first estimates the interval time of training ever-waiting links using the Kaplan-Meier (KM) (Kaplan and Meier, 1958) estimation method and then by using all parasocial links from training period to train a linear model. This linear model can be trained through MLE as described above. For more practical use, Wang et al. (Wang and Wang, 2010) proposed twin boosting method with BJ estimator, we use this method to solve the RLTP problem.

Accelerated Failure Time (AFT) model: An AFT model assumes that the logarithm of the interval time $\log (\boldsymbol{T})$ follows a statistical distribution and it is linearly related to the (topological) feature vectors. The general form for AFT regression model is

$$
\log (\boldsymbol{T})=\boldsymbol{X} \cdot \boldsymbol{\beta}+\sigma \cdot \epsilon
$$

where $\boldsymbol{X}$ is the covariate matrix of size $N \times d$ where $i^{\text {th }}$ row of $\boldsymbol{X}$ is $\mathbf{x}_{i}, \boldsymbol{\beta}$ is a $d$ dimensional coefficient vector (model parameters), $\sigma(\sigma>0)$ is an unknown scale parameter, and $\epsilon$ is an error variable which follows a similar distribution 
Table 4: Density, Survival and Hazard functions for the distributions used with AFT model. $\lambda$ is scale parameter and $k$ is shape parameter for both Weibull and $\log$-logistic distribution. For log-normal distribution $\mu$ is the mean (location parameter), $\sigma^{2}$ is the variance and $\Phi$ is cumulative distribution function of normal distribution.

\begin{tabular}{lccc}
\hline Distributions & Density Function & Survival Function & Hazard Function \\
\hline Weibull & $\lambda k t^{k-1} \cdot \exp \left(-\lambda t^{k}\right)$ & $\exp \left(-\lambda t^{k}\right)$ & $\lambda k t^{k-1}$ \\
Log-Normal & $\frac{1}{\sqrt{2 \pi} \sigma t} \exp \left(-\frac{(\log (t)-\mu)^{2}}{2 \sigma^{2}}\right)$ & $1-\Phi\left(\frac{\log (t)-\mu}{\sigma}\right)$ & $\frac{1}{\sqrt{2 \pi} \sigma t} \exp \left(-\frac{(\log (t)-\mu)^{2}}{2 \sigma^{2}}\right)$ \\
Log-Logistic & $\frac{\lambda k t^{k-1}}{\left(1+\lambda t^{k}\right)^{2}}$ & $\frac{1}{1+\lambda t^{k}}$ & $\frac{\lambda k t^{k-1}}{1+\lambda t^{k}}$ \\
\hline
\end{tabular}

to $\log (\boldsymbol{T})$. For our problem, we use the three most suitable distributions (see Figure 3) for interval time, the details of which are given in Table 4.

\subsection{Algorithmic framework}

In Algorithm 1, we describe a general framework of our proposed method. First we divide the time-stamps of the input graph into train and test periods as mentioned in line 1 of Algorithm 1. After that we create training data instances (train-set) and test data instance (test-set) from the corresponding train and test periods (Lines 2-4). Then we calculate topological features for each parasocial link (data instance) in the train-set and test-set as described in Lines 5-10 of Algorithm 1. After that we generate target variable for each data instance (Lines 11-26), for which we observe the corresponding reciprocal link in the graph. For a parasocial link $e \in$ train-set, if the corresponding reciprocal link is generated during train period then interval time Int(e) (Section 3.1 ) is the target value with event indicator value $C_{e}=1$ otherwise time difference between the link creation and end of training period act as the target value with event indicator value $C_{e}=0$. Similarly, we generate target values for data instance of test-set as explained in Lines 27-35 of Algorithm 1. Then, we use $R$ libraries to train the survival models with training data and predict target values for the test data to generate the test results (test-res) and lastly we evaluate that test-res.

\section{Experiments and Results}

We conducted a set of rigorous experiments to demonstrate the benefit of using censored information and the superiority of proposed survival models to solve the RLTP problem. We used five proposed survival models: Cox regression model, AFT model with Weibull, log-normal and log-logistic distributions, and Buckley-James (BJ) regression model. To prove the fact that the proposed survival models are better suited for solving the RLTP problem, we compared 


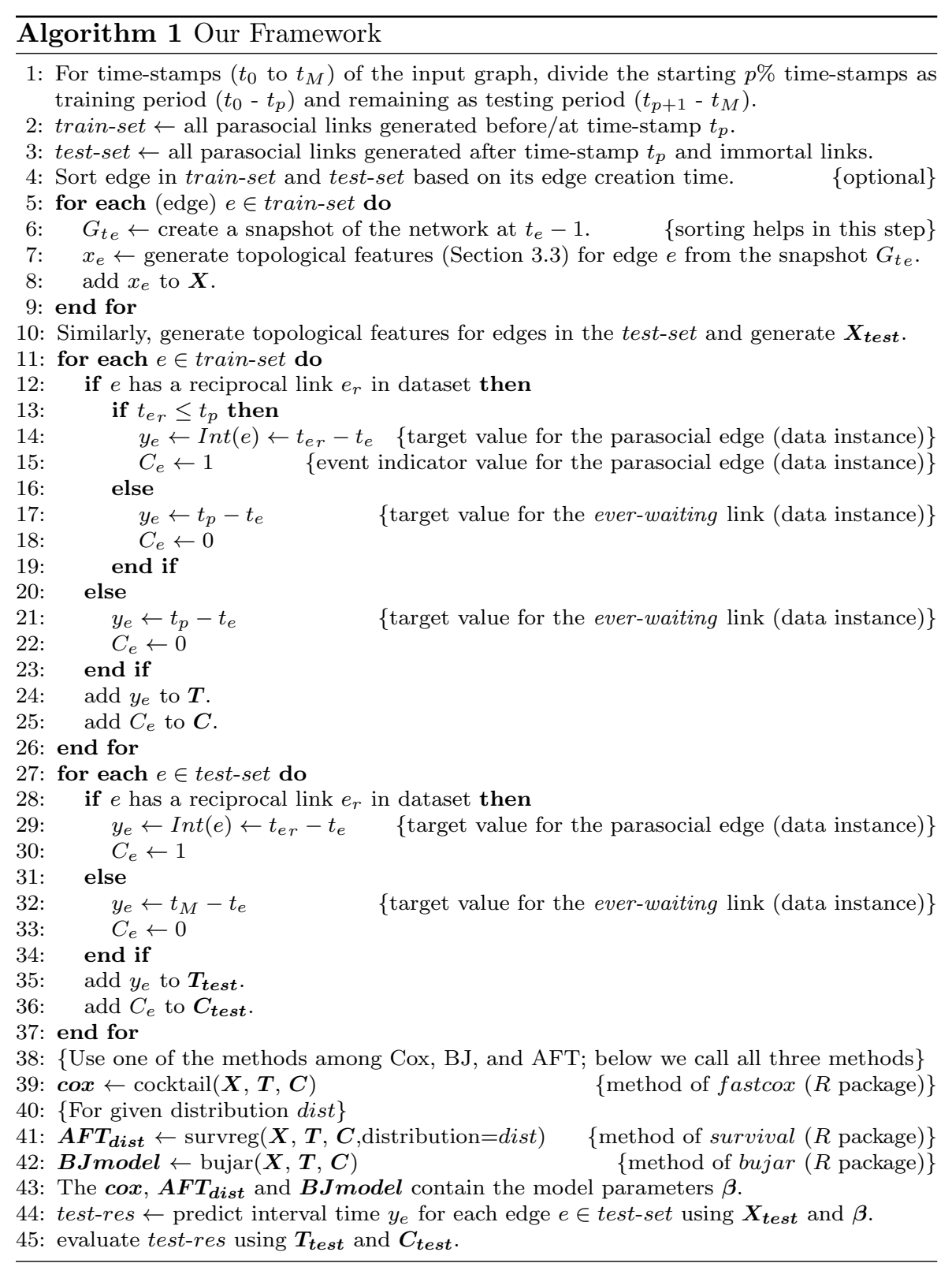

them with traditional regression models such as ridge regression (RidgeReg), lasso regression (LassoReg), feed forward neural networks (FFNN) and support vector regression (SVR). Note that these traditional regression models cannot use censored information (ever-waiting links). We also compare proposed Cox regression model with generalized linear model (GML), which is an adopted model from (Sun et al, 2012). 
In addition to the suitability of the proposed survival models for the RLTP problem, we also demonstrate the usability of the ever-waiting links. For that, we conducted experiments where we train the survival models without censored information and compare the performance of the models on the test dataset. We report the improvement in the performance when the ever-waiting links are used for training the survival models.

Lastly, we conduct an experiment to show that reciprocal links with short interval time contain enough information required for training the survival models.

\subsection{Datasets}

For the experiments, we use three real world datasets Epinion, MC-Email and Enron. We discuss these datasets in Section 3.2 and basic statistics of the datasets are shown in Table 1.

Generating a synthetic dataset for the RLTP problem is a challenging task, because in the literature most of the synthetic graph generation models try to mimic basic real-world properties such as power-law degree distribution (Faloutsos et al, 1999), community structures (Leskovec et al, 2007), etc. All these methods generate directed networks with extremely low reciprocitygenerally, less than 1\%. Durak et al. have proposed a synthetic network generation algorithm which also considers reciprocity (Nurcan Durak, 2013). We use this algorithm for generating three synthetic graphs where the vertex count varies between $10,000(10 K)$ to $30,000(30 K)$ with increments of $10 K$. Edges of these synthetic networks have no time-stamps; hence, we assign random time-stamps between 0 to 100 to parasocial links. The time-stamps of reciprocal links of these synthetic networks are selected by matching the reciprocal link interval time of the Epinion dataset through the best fit Weibull distribution.

\subsection{Experimental Setting}

For our experiments, we divide the time-stamps of a dataset into two nonoverlapping continuous partitions, where the earlier partition is the train period and the latter is the test period. In three different experiments, we use, respectively, $60 \%, 70 \%$ and $80 \%$ of the earlier time-stamps as the train periods and the remaining time-stamps as the test period. For synthetic datasets, a 70:30 split of the time-stamps is used as the train and test period of our experiments. For calculating the topological features explained in Section 3.3 for a parasocial link (data instance), we take a snapshot of the network until the time-stamp of the corresponding reciprocal link or end of the train period (whichever is earlier).

Like any other link prediction task, RLTP also suffers from the class imbalance issue, where the number of positive instances $\left(C_{i}=1\right)$ is much smaller 
than that of the negative instances. To alleviate this problem we use the wellknown majority undersampling (Bunkhumpornpat et al, 2011) strategy as discussed below: all the reciprocal links generated during a train period are considered in the training data pool as positive instances and only $50 \%$ of the parasocial links generated during the same period are censored negative instances $\left(C_{i}=0\right)$ in the pool. The test data pool (and their labels) are also generated similarly from the test period. As train and test data instances need to be from their corresponding time periods, we use a modified K-fold cross validation, where each fold contains a random subset of train and test data instances from their respective pools. For all our experiments, we used 5-fold cross validation in this manner.

For minimizing the objective function (Equation (4)) of censored problem formulation of RLTP, for the Cox regression model, we used cocktail algorithm (Yang and Zou, 2013) (the library is provided by the authors of (Yang and Zou, 2013)). For AFT models and BJ regression, we used Survival package $^{5}$ and Bujar package ${ }^{6}$, respectively, available in R. For RidgeReg, LassoReg and SVR, we used scikit-learn python library and for FFNN, we used Matlab NNtoolbox. We used TopCom indexing method (Dave and Hasan, $2015,2016)$ to find shortest directed distance feature. To choose the best parameters of SVR, we used grid search, where the cost parameter $C$ takes values from $\{0.0001,0.001,0.01,0.1,1.0\}$ and Epsilon $(\epsilon)$ takes values from $\{0.0001,0.001,0.01,1.0\}$.

\subsection{Evaluation Metrics}

Datasets generated from directed time-stamped networks are longitudinal data and for the RLTP problem the datasets also contain censored information. Evaluating models on these datasets using traditional evaluation metrics is not suitable, instead we use time-dependent AUC (also known as c-Index), which is widely used in longitudinal data analysis (Pencina and D'Agostino, 2004).

For a pair of data instances, assume $\left(y_{i}, y_{j}\right)$ and $\left(\widehat{y}_{i}, \widehat{y}_{j}\right)$ are the target and the predicted values, respectively. The time-dependent AUC is defined as the probability of $\widehat{y}_{i}>\widehat{y}_{j}$ given $y_{i}>y_{j}$. If target $y_{i}$ has only 2 possible values, then time-dependent AUC is the same as the popular AUC (Area Under ROC Curve) metric for classification. Similar to the AUC metric, time-dependent AUC takes values between 0 to 1 , where 1 is the best possible value for this metric. Time-dependent AUC (TD-AUC) is calculated as follows:

$$
T D-A U C=\frac{1}{N_{c n t}} \sum_{i: C_{i}=1} \sum_{y_{j}>y_{i}} \mathbb{1}\left(\widehat{y}_{j}>\widehat{y_{i}}\right)
$$

where, $N_{c n t}$ is total count of $\left(y_{i}, y_{j}\right)$ pairs such that $C_{i}=1$ (the event has occurred) and $y_{j}>y_{i}$ holds.

\footnotetext{
5 cran.r-project.org/package=survival

6 cran.r-project.org/web/packages/bujar/index.html
} 
For the Cox regression model, the predicted value is the hazard value and for a higher hazard value the event occurs earlier, hence the time-dependent AUC for Cox can be calculated as:

$$
T D-A U C=\frac{1}{N_{c n t}} \sum_{i: C_{i}=1} \sum_{y_{j}>y_{i}} \mathbb{1}\left(\mathbf{x}_{i}^{T} \widehat{\boldsymbol{\beta}}>\mathbf{x}_{j}^{T} \widehat{\boldsymbol{\beta}}\right)
$$

4.4 Comparison results of survival models and regression models

We compared proposed survival models with four other traditional regression models and our results are shown in Tables 5, 6 and 7, where columns represent different training splits and each row represents a prediction model. A horizontal bar separates the traditional regression models in the upper part and the survival based models in the lower part. Here, each table cell shows mean and standard deviation for TD-AUC values. For most of the cases, the Cox regression model performs the best.

For the Epinion dataset, as depicted in Table 5, the Cox regression model performs the best with mean TD-AUC $0.7364,0.7463$ and 0.7485 for training period with $60 \%, 70 \%$ and $80 \%$ splits of time-stamps, respectively. Here, with increase in the training data we can clearly see improvement in the performance, which is an expected behavior because with more training examples the model learns better. BJ model is the next best with performance very close to the Cox model. For this model also, the mean TD-AUC improves from 0.7312 to 0.7416 as we increase the training data. Similar behavior is observed for other survival models, but the performance of the AFT models is, unfortunately, not good for the dataset. This can be attributed to the fact that AFT models make strict distribution assumptions on the data and such assumption may not be suitable for the Epinion dataset (Figure 3).

For the Epinion dataset, among the traditional regression based methods, ridge regression performs better than any other competing methods with mean TD-AUC in the range between 0.60 and 0.61 . But, when we compare its performance over different training splits, we see that its performance does not

Table 5: Epinion Dataset: TD-AUC results [mean ( \pm standard deviation)] with different splits used for training period.

\begin{tabular}{lccc}
\hline Method / Split & $60 \%$ & $70 \%$ & $80 \%$ \\
\hline RidgeReg & $0.6185( \pm .0018)$ & $0.6086( \pm .0013)$ & $0.6060( \pm .0018)$ \\
LassoReg & $0.6169( \pm .0013)$ & $0.6020( \pm .0014)$ & $0.6039( \pm .0017)$ \\
FFNN & $0.5510( \pm .1296)$ & $0.5048( \pm .0822)$ & $0.4456( \pm .0725)$ \\
SVR & $0.4791( \pm .0005)$ & $0.4871( \pm .0039)$ & $0.4914( \pm .0030)$ \\
\hline BJ Model & $0.7312( \pm .0010)$ & $0.7339( \pm .0020)$ & $0.7416( \pm .0021)$ \\
Weibull & $0.3807( \pm .0763)$ & $0.5210( \pm .1446)$ & $0.5232( \pm .1282)$ \\
logNormal & $0.3660( \pm .0388)$ & $0.4461( \pm .0283)$ & $0.4283( \pm .0305)$ \\
logLogistic & $0.4901( \pm .0098)$ & $0.5110( \pm .0196)$ & $0.5132( \pm .0188)$ \\
Cox & $\mathbf{0 . 7 3 6 4}( \pm .0025)$ & $\mathbf{0 . 7 4 3 6}( \pm .0016)$ & $\mathbf{0 . 7 4 8 5}( \pm .0028)$ \\
\hline
\end{tabular}


Table 6: $M C$-Email Dataset: TD-AUC results [mean ( \pm standard deviation)] with different splits used for training period.

\begin{tabular}{lccc}
\hline Method / Split & $60 \%$ & $70 \%$ & $80 \%$ \\
\hline RidgeReg & $0.6213( \pm .0087)$ & $0.6083( \pm .0146)$ & $0.6014( \pm .0125)$ \\
LassoReg & $0.5884( \pm .0100)$ & $0.5709( \pm .0201)$ & $0.5686( \pm .0074)$ \\
FFNN & $0.4199( \pm .0800)$ & $0.4609( \pm .0964)$ & $0.5069( \pm .0915)$ \\
SVR & $0.5462( \pm .0154)$ & $0.5737( \pm .0187)$ & $0.5530( \pm .0150)$ \\
\hline BJ Model & $0.5898( \pm .0087)$ & $0.5910( \pm .0146)$ & $0.6103( \pm .0059)$ \\
Weibull & $0.6139( \pm .0075)$ & $0.6171( \pm .0069)$ & $0.6315( \pm .0166)$ \\
logNormal & $0.6391( \pm .0053)$ & $0.6463( \pm .0015)$ & $0.6695( \pm .0116)$ \\
logLogistic & $0.6380( \pm .0121)$ & $0.6494( \pm .0062)$ & $0.6747( \pm .0201)$ \\
Cox & $\mathbf{0 . 6 5 2 7}( \pm .0097)$ & $\mathbf{0 . 6 5 5 8}( \pm .0125)$ & $\mathbf{0 . 6 7 9 7}( \pm .0062)$ \\
\hline
\end{tabular}

Table 7: Enron Dataset: TD-AUC results [mean ( \pm standard deviation)] with different splits used for training period.

\begin{tabular}{lccc}
\hline Method / Split & $60 \%$ & $70 \%$ & $80 \%$ \\
\hline RidgeReg & $0.5732( \pm .0073)$ & $0.5847( \pm .0159)$ & $0.5318( \pm .0164)$ \\
LassoReg & $0.5740( \pm .0076)$ & $0.5850( \pm .0152)$ & $0.5309( \pm .0178)$ \\
FFNN & $0.4900( \pm .0258)$ & $0.5407( \pm .0434)$ & $0.5363( \pm .0561)$ \\
SVR & $0.5490( \pm .0080)$ & $0.5680( \pm .0176)$ & $0.5608( \pm .0136)$ \\
\hline BJ Model & $0.5292( \pm .0120)$ & $0.6096( \pm .0076)$ & $0.5599( \pm .0121)$ \\
Weibull & $0.5710( \pm .0168)$ & $\mathbf{0 . 6 3 1 9}( \pm .0050)$ & $\mathbf{0 . 5 9 8 0}( \pm .0096)$ \\
logNormal & $0.5713( \pm .0146)$ & $0.6146( \pm .0097)$ & $0.5862( \pm .0129)$ \\
logLogistic & $0.5787( \pm .0171)$ & $0.6224( \pm .0069)$ & $0.5917( \pm .0101)$ \\
Cox & $\mathbf{0 . 5 8 5 4 ( \pm . 0 1 6 6 )}$ & $0.6311( \pm .0110)$ & $0.5919( \pm .0084)$ \\
\hline
\end{tabular}

improve as we increase the training data. The same behavior holds for other traditional regression methods, such as Lasso regression and FFNN. One possible explanation for this behavior is model under-fitting; that is, the majority of the errors in the traditional regression models are coming from the bias error, so the error does not improve much with a larger dataset which reduces variance error only. On the other hand, survival analysis based models are more sophisticated, which enables them to design complex functions for predicting the time, thus overcoming the under-fitting issue.

For the $M C$-Email dataset, the overall behavior of the models is very similar to the Epinion dataset. Here again the Cox regression model performs the best with mean TD-AUC between 0.65 and 0.68 and its results are improved for larger training data. Performance of different AFT models vary, but they all perform better than all of the traditional regression methods. In particular, AFT with log-logistic and log-normal distributions perform great and their mean TD-AUC is very close to the results of the Cox regression as shown in Table 6 . The performance of all survival models improve as we provide more training data. On the other hand, best among the competing methods is ridge regression with a mean TD-AUC between 0.60 and 0.62 . As we have discussed earlier, this model suffers from under-fitting problem. 
Table 8: TD-AUC results [mean ( \pm standard deviation)] for various methods on synthetic datasets.

\begin{tabular}{lccc}
\hline Method & $10 \mathrm{~K}$ & $20 \mathrm{~K}$ & $30 \mathrm{~K}$ \\
\hline RidgeReg & $0.5210( \pm .0029)$ & $0.4949( \pm .0018)$ & $0.5203( \pm .0023)$ \\
LassoReg & $0.5150( \pm .0034)$ & $0.4876( \pm .0059)$ & $0.5091( \pm .0048)$ \\
FFNN & $0.4999( \pm .0517)$ & $0.4967( \pm .0151)$ & $0.5068( \pm .0631)$ \\
SVR & $0.5379( \pm .0021)$ & $0.4963( \pm .0026)$ & $0.5473( \pm .0015)$ \\
\hline BJ Model & $0.5589( \pm .0011)$ & $0.5232( \pm .0013)$ & $0.5557( \pm .0008)$ \\
Weibull & $0.5641( \pm .0036)$ & $0.4954( \pm .0027)$ & $0.5559( \pm .0015)$ \\
logNormal & $\mathbf{0 . 5 6 7 0}( \pm .0027)$ & $0.4991( \pm .0030)$ & $\mathbf{0 . 5 6 1 8}( \pm .0011)$ \\
logLogistic & $0.5597( \pm .0029)$ & $0.4985( \pm .0042)$ & $0.5576( \pm .0019)$ \\
Cox & $0.5604( \pm .0025)$ & $\mathbf{0 . 5 2 8 2}( \pm .0026)$ & $0.5558( \pm .0016)$ \\
\hline
\end{tabular}

For the Enron dataset, results are shown in Table 7. Here, for the training period with $60 \%$ split, Cox regression performs the best with mean TD-AUC 0.58. For the other two splits, AFT model with Weibull distribution performs the best with mean TD-AUC 0.63 and 0.59. The BJ model performs poorly compared to the other survival models with mean TD-AUC ranging from 0.52 to 0.6 , but the performance of BJ model is still better than all competing regression methods for training period with $70 \%$ and $80 \%$ splits of time-stamps. For this dataset, for $80 \%$ training split, none of the models have better performance than the other splits. This is due to the fact that this dataset is extremely sparse and it has only 3,007 links created during 944 time-stamps (Table 1). Hence even the $80 \%$ split does not provide more informative training samples to perform good prediction on remaining data.

The results for synthetic networks are shown in the Table 8 by using the mean TD-AUC and standard deviation metrics. As we observe the results in this table, We can easily conclude that survival models always perform better than traditional regression methods. For two datasets with $10 K$ and $30 K$ node instances, the AFT model with log-normal distribution performs the best among all, while for the dataset with $20 K$ nodes the Cox regression performs the best. The performance of survival models is consistently very similar except for dataset with $20 K$ node where Cox and BJ models clearly perform better than AFT models. Among competing methods, SVR always performs better than others.

\subsubsection{Comparison with GLM}

Sun et al. (Sun et al, 2012) proposed a method to predict link generation time in a heterogeneous network, where they design a unique feature for the task and use the feature with generalized linear model (GLM) for the prediction task. This proposed feature is designed based on meta-path (a simple path with link label information) in a heterogeneous network. We adopted this feature for a homogeneous network and the adopted feature can be described as a number of simple paths of size $k$ between two nodes. Counting the number 


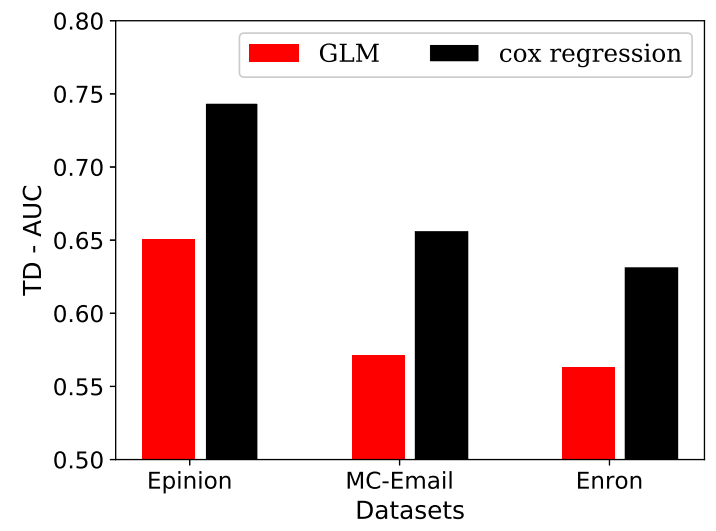

Fig. 6: Comparison of GLM and cox regression

of simple paths is an extremely costly operation especially for a large dataset such as the Epinion network; hence for this experiment, we use $k$ upto 5 i.e. $k \in\{2,3,4,5\}$ for all three networks, Epinion, MC-Email and Enron. We provide these homogeneous feature values to GLM (with gamma distribution) to solve the RLTP problem. For this experiment we use a $70 \%$ split of timestamps as train period and remaining $30 \%$ as test period. The results of this experiment are depicted in Figure 6, where GLM is compared with the Cox regression model for all three datasets. From Figure 6, we observe that the Cox regression outperforms the GLM model for all three datasets by noticeable margins. We believe, one of the main reasons for the poor performance of the GLM based method is that the feature proposed by Sun et al (2012) is carefully designed for an author-paper based heterogeneous network and its adoption in a homogeneous network is not very useful.

\subsection{Importance of ever-waiting links}

We conducted experiments to show the importance of ever-waiting links and the results are depicted in Tables 9 and 10. Table 9 shows the increment in TD-AUC up to $62 \%$ in the real-world datasets, when the survival models are provided with censored information (ever-waiting links) during the training, as compared to when the models are trained without censored information. For the Epinion dataset, the increment in the results is significant (more than $27 \%$ for all models) except AFT with log-normal distribution. Similarly, for the MC-Email and the Enron datasets the increment is up to $27 \%$, which is substantial. As shown in Table 10, for the synthetic datasets we also have very similar increment in the results except for the BJ model with datasets of $10 \mathrm{~K}$ nodes. For the most part the increment in performance is high for the Cox regression and the AFT with Weibull distribution. However, for other models the increment is limited to around $10 \%$. The modest contribution of 
Table 9: Time-Dependent AUC results [mean ( \pm standard deviation)] for survival analysis methods with and without ever-waiting Links on real datasets.

\begin{tabular}{lccc}
\hline \multicolumn{5}{c}{ Epinion } \\
\hline model & w/o ever-waiting & with ever-waiting & $\%$ incr \\
\hline BJ Model & $0.4580( \pm .0042)$ & $0.7416( \pm .0021)$ & 61.94 \\
Weibull & $0.4096( \pm .0090)$ & $0.5232( \pm .1282)$ & 27.73 \\
logNormal & $0.4218( \pm .0035)$ & $0.4283( \pm .0305)$ & 1.53 \\
logLogistic & $0.3767( \pm .0024)$ & $0.5132( \pm .0188)$ & 36.23 \\
Cox & $0.4975( \pm .0024)$ & $0.7485( \pm .0028)$ & 50.45 \\
\hline \multicolumn{5}{c}{ MC-Email } \\
\hline model & w/o ever-waiting & with ever-waiting & $\%$ incr \\
\hline BJ Model & $0.4787( \pm .0131)$ & $0.6103( \pm .0059)$ & 27.51 \\
Weibull & $0.5517( \pm .0101)$ & $0.6315( \pm .0166)$ & 14.46 \\
logNormal & $0.6342( \pm .0146)$ & $0.6695( \pm .0116)$ & 5.56 \\
logLogistic & $0.6331( \pm .0152)$ & $0.6747( \pm .0201)$ & 6.57 \\
Cox & $0.6102( \pm .0137)$ & $0.6797( \pm .0062)$ & 11.38 \\
\hline \multicolumn{5}{c}{ Enron } \\
\hline model & w/o ever-waiting & with ever-waiting & $\%$ incr \\
\hline BJ Model & $0.5499( \pm .0134)$ & $0.5599( \pm .0121)$ & 1.82 \\
Weibull & $0.5330( \pm .0237)$ & $0.5980( \pm .0096)$ & 12.20 \\
logNormal & $0.5344( \pm .0070)$ & $0.5862( \pm .0129)$ & 9.71 \\
logLogistic & $0.5379( \pm .0053)$ & $0.5917( \pm .0101)$ & 10.01 \\
Cox & $0.5481( \pm .0234)$ & $0.5919( \pm .0084)$ & 7.99 \\
\hline
\end{tabular}

Table 10: Time-Dependent AUC results [mean ( \pm standard deviation)] for survival analysis methods with and without ever-waiting Links on synthetic datasets.

\begin{tabular}{lccc}
\hline \multicolumn{5}{c}{ 10K } \\
\hline model & w/o ever-waiting & with ever-waiting & \%incr \\
\hline BJ Model & $0.5730( \pm .0045)$ & $0.5589( \pm .0011)$ & -2.46 \\
Weibull & $0.4847( \pm .0096)$ & $0.5641( \pm .0036)$ & 16.37 \\
logNormal & $0.5564( \pm .0102)$ & $0.5670( \pm .0027)$ & 1.89 \\
logLogistic & $0.5546( \pm .0128)$ & $0.5597( \pm .0029)$ & 0.92 \\
Cox & $0.4910( \pm .0037)$ & $0.5604( \pm .0025)$ & 14.14 \\
\hline \multicolumn{5}{c}{$\mathbf{2 0 K}$} \\
\hline model & w/o ever-waiting & with ever-waiting & $\%$ incr \\
\hline BJ Model & $0.4956( \pm .0062)$ & $0.5232( \pm .0013)$ & 5.57 \\
Weibull & $0.4951( \pm .0025)$ & $0.4954( \pm .0027)$ & 0.06 \\
logNormal & $0.4984( \pm .0018)$ & $0.4991( \pm .0030)$ & 0.15 \\
logLogistic & $0.4965( \pm .0055)$ & $0.4985( \pm .0042)$ & 0.41 \\
Cox & $0.4938( \pm .0098)$ & $0.5282( \pm .0026)$ & 6.97 \\
\hline \multicolumn{4}{c}{$\mathbf{3 0 K}$} \\
\hline model & w/o ever-waiting & with ever-waiting & $\%$ incr \\
\hline BJ Model & $0.5548( \pm .0049)$ & $0.5557( \pm .0008)$ & 0.17 \\
Weibull & $0.4544( \pm .0020)$ & $0.5559( \pm .0015)$ & 22.35 \\
logNormal & $0.5270( \pm .0044)$ & $0.5618( \pm .0011)$ & 6.61 \\
logLogistic & $0.5243( \pm .0042)$ & $0.5576( \pm .0019)$ & 6.35 \\
Cox & $0.4637( \pm .0051)$ & $0.5558( \pm .0016)$ & 19.86 \\
\hline
\end{tabular}

ever-waiting links for the case of synthetic networks can be attributed to the network generation model. We used Durak et al's model (Nurcan Durak, 2013), which selects pairs of vertices for reciprocal link creation based on only degree distribution without considering any of the social phenomena, so the features that we are using may be not very effective for the synthetic datasets. 


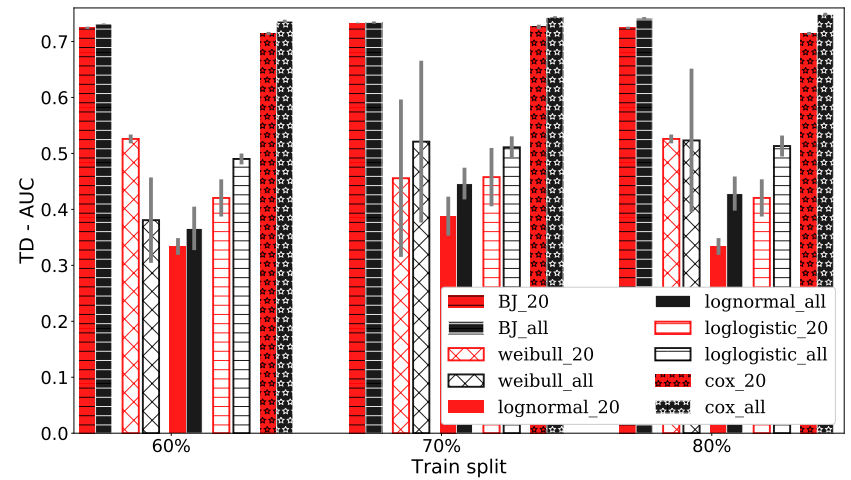

Fig. 7: Epinion Dataset: Comparison of training with top $20 \%$ reciprocal links and all reciprocal links.

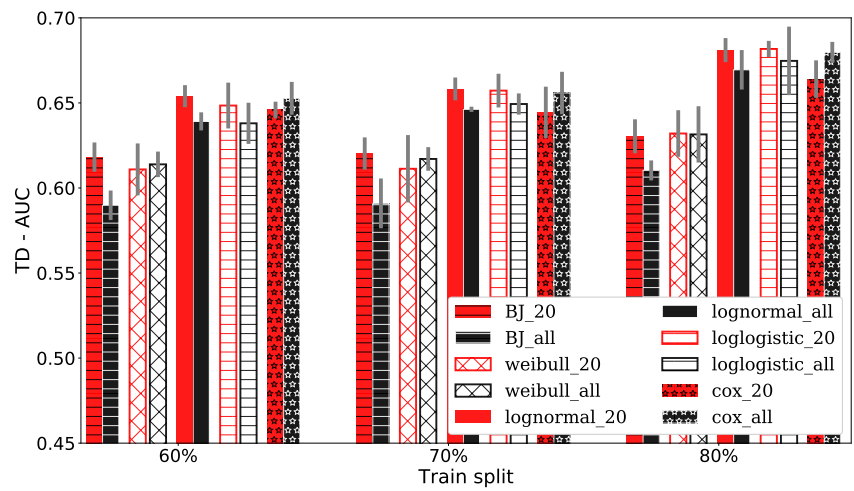

Fig. 8: MC-Email Dataset: Comparison of training with top 20\% reciprocal links and all reciprocal links.

4.6 Importance of reciprocal links with small interval time

For the RLTP problem, reciprocal links carry very useful information and this information is not distributed uniformly over all reciprocal links. We described in Section 3.2 that for most of the reciprocal links the corresponding time interval is relatively small, and very few have high time interval as depicted in Figure 2. The reciprocal links for which the corresponding time interval is equal to or less than $20 \%$ of the maximum time interval among all the time intervals of reciprocal links in the dataset are called "top 20\%" reciprocal links. We trained survival models with top $20 \%$ reciprocal links (with ever-waiting 


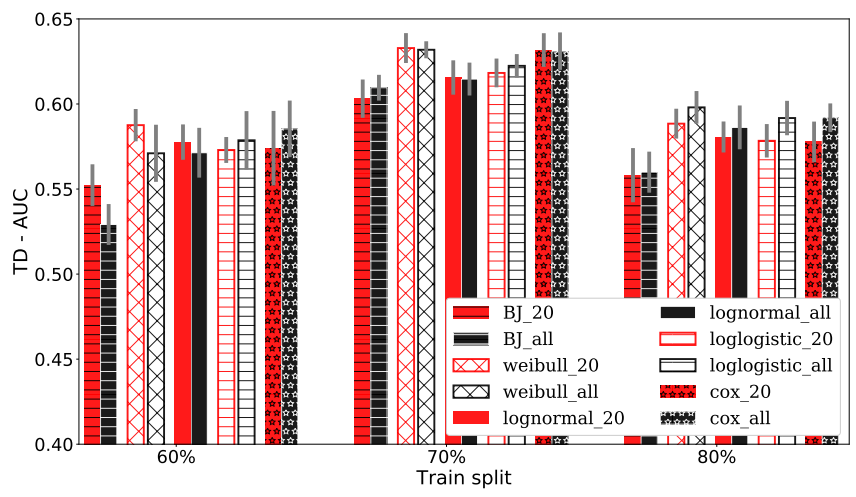

Fig. 9: Enron Dataset: Comparison of training with top 20\% reciprocal links and all reciprocal links.

links) and compared the results of these models with results of models trained with all reciprocal links (with ever-waiting links).

Results for these experiments are shown in Figures 7, 8, 9, where all red bars represent different models trained with top 20\% reciprocal links and all black bars represent the same models trained using all reciprocal links. We can see that, for all three datasets, survival models trained with top $20 \%$ reciprocal links perform very similar or better to the models trained with all reciprocal links. This observation supports our argument that all reciprocal links do not carry same amount of information, but notable amounts of information lie in the reciprocal links with short interval time.

\subsection{Contribution of Top-5 features}

In Section 3.3.3, we study correlation of different features with interval time. Through this experiment, we study the contribution of top five highly correlated features (top5-features) to solve the RLTP problem. From the Table 2, we can find these top5-features for each real-world dataset. We can see, for

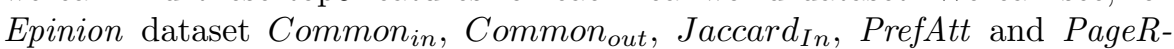
ank(u) are highly correlated features. Similarly, for both $M C$-Email and En-

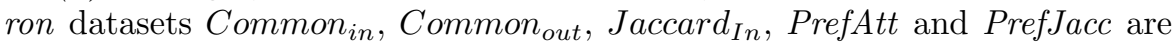
the top5-features (Table 2). For this comparison study, we prepared train and test instances similarly as described in Section 4.2 with $70 \%$ training split, but the difference is, here each data instance is represented by only corresponding top5-features. We use proposed survival models (Section 3.5) with top5-features data to solve the RLTP problem.

Table 11 shows results for the comparison experiment with mean TDAUC value and standard deviation for 5 independent runs. The last column 
Table 11: Time-Dependent AUC results [mean ( \pm standard deviation)] for survival analysis methods with top5-features and all features.

\begin{tabular}{lccc}
\hline \multicolumn{5}{c}{ Epinion } \\
\hline model & top5-features & all-features & $\%$ incr \\
\hline BJ Model & $0.6541( \pm .0027)$ & $0.7339( \pm .0020)$ & 12.20 \\
Weibull & $0.4878( \pm .0872)$ & $0.5210( \pm .1446)$ & 6.80 \\
logNormal & $0.3157( \pm .0062)$ & $0.4461( \pm .0283)$ & 41.32 \\
logLogistic & $0.3360( \pm .0032)$ & $0.5110( \pm .0196)$ & 52.10 \\
Cox & $0.6292( \pm .0056)$ & $0.7436( \pm .0016)$ & 18.19 \\
\hline \multicolumn{5}{c}{ MC-Email } \\
\hline model & top5-features & all-features & $\%$ incr \\
\hline BJ Model & $0.4728( \pm .0059)$ & $0.5910( \pm .0146)$ & 25.00 \\
Weibull & $0.5503( \pm .0102)$ & $0.6171( \pm .0069)$ & 12.13 \\
logNormal & $0.5802( \pm .0074)$ & $0.6463( \pm .0015)$ & 11.40 \\
logLogistic & $0.5917( \pm .0125)$ & $0.6494( \pm .0062)$ & 9.76 \\
Cox & $0.5738( \pm .0187)$ & $0.6558( \pm .0125)$ & 14.29 \\
\hline \multicolumn{4}{c}{ Enron } \\
\hline model & top5-features & all-features & $\%$ incr \\
\hline J Model & $0.5995( \pm .0061)$ & $0.6096( \pm .0076)$ & 1.69 \\
Weibull & $0.5985( \pm .0140)$ & $0.6319( \pm .0050)$ & 5.58 \\
logNormal & $0.5972( \pm .0130)$ & $0.6146( \pm .0097)$ & 2.92 \\
logLogistic & $0.6043( \pm .0070)$ & $0.6224( \pm .0069)$ & 2.99 \\
Cox & $0.5964( \pm .0069)$ & $0.6311( \pm .0110)$ & 5.82 \\
\hline
\end{tabular}

in the Table 11 shows the increment in the mean TD-AUC value from top5features data to all features data. This table clarifies the importance of the other features with lower correlation values (Table 2), because for both the Epinion and the $M C$-Email datasets the increment in the results is noticeable. But for the Enron dataset the increment is not very impressive; we believe low number of data instances and very high correlation of top5-features are the main reasons for this shortcoming.

\section{Conclusion and Future Works}

In this paper, we proposed a novel problem, namely, reciprocal link time prediction $(R L T P)$, which has wide applicability in email, social and other directed networks. We designed various socially meaningful topological features specifically for directed networks, which are useful to solve the RLTP problem. We mapped the RLTP problem into a survival analysis task and through experiments on three real-life network datasets, we showed that such a framework is better suited than traditional regression based approaches for solving the RLTP problem. We demonstrated that using ever-waiting links for training adds valuable information to the prediction models. We also investigated the information contributed by the reciprocal links and show that the majority of the required information lies in the top few percent $(20 \%)$ of the reciprocal links. To the best of our knowledge this is the first study on time interval prediction for reciprocal links, which is useful to answer response time for emails or friend requests. It can also be used for recommendation in trust networks for 
suggesting a new connection (parasocial link) for which, the predicted response time is very small.

The RLTP is a novel problem and this is one of the earliest and comprehensive study of the problem. There do exist some opportunities to extend our work. For example, we have used basic survival analysis based regression models, but one can study the timing patterns and design complex regression model by considering the timing patterns. Also, one can design sophisticated time dependent topological features that carry more information to solve the RLTP problem and study different sparse prediction models to find suitable features for the prediction.

Acknowledgements This research is supported by National Science Foundation (NSF) career award (IIS-1149851) and in part by the NSF grants IIS-1707498, IIS-1619028 and IIS-1646881

\section{References}

Adalı S, Golbeck J (2014) Predicting personality with social behavior: a comparative study. Social Network Analysis and Mining 4(1):159

Akaike H (1998) Information Theory and an Extension of the Maximum Likelihood Principle, Springer New York, New York, NY, pp 199-213

Anand S, Chandramouli R, Subbalakshmi KP, Venkataraman M (2013) Altruism in social networks: good guys do finish first. Social Network Analysis and Mining 3(2):167-177

Bogdanov P, Busch M, Moehlis J, Singh AK, Szymanski BK (2014) Modeling individual topic-specific behavior and influence backbone networks in social media. Social Network Analysis and Mining 4(1):204

Bunkhumpornpat C, Sinapiromsaran K, Lursinsap C (2011) Mute: Majority under-sampling technique. In: International Conference on Information, Communications and Signal Processing (ICICS), IEEE, pp 1-4

Cheng J, Romero DM, Meeder B, Kleinberg J (2011) Predicting reciprocity in social networks. In: Privacy, Security, Risk and Trust (PASSAT) and 2011 IEEE Third Inernational Conference on Social Computing (SocialCom), 2011 IEEE Third International Conference on, pp 49-56

Chierichetti F, Kumar R, Lattanzi S, Mitzenmacher M, Panconesi A, Raghavan P (2009) On compressing social networks. In: ACM SIGKDD international conference on Knowledge discovery and data mining, pp 219-228

Cox DR (1972) Regression models and life-tables. Journal of the Royal Statistical Society Series B (Methodological) 34(2):187-220

Dave VS, Hasan MA (2015) Topcom: Index for shortest distance query in directed graph. In: Database and Expert Systems Applications, Springer International Publishing, Cham, pp 471-480

Dave VS, Hasan MA (2016) Topcom: Index for shortest distance query in directed graph. CoRR abs/1602.01537, URL http://arxiv.org/abs/1602. 01537 
Dave VS, Al Hasan M, Reddy CK (2017) How fast will you get a response? predicting interval time for reciprocal link creation. In: AAAI International Conference on Web and Social Media (ICWSM)

Devineni P, Koutra D, Faloutsos M, Faloutsos C (2017) Facebook wall posts: a model of user behaviors. Social Network Analysis and Mining 7(1):6

Dong X, Gabrilovich E, Heitz G, Horn W, Lao N, Murphy K, Strohmann T, Sun S, Zhang W (2014) Knowledge vault: A web-scale approach to probabilistic knowledge fusion. In: Proceedings of the 20th ACM SIGKDD International Conference on Knowledge Discovery and Data Mining, KDD '14, pp 601-610

Dong Y, Tang J, Wu S, Tian J, Chawla NV, Rao J, Cao H (2012) Link prediction and recommendation across heterogeneous social networks. In: Proceedings of the 2012 IEEE 12th International Conference on Data Mining, ICDM '12, pp 181-190

Dumba B, Golnari G, Zhang ZL (2016) Analysis of a Reciprocal Network Using Google+: Structural Properties and Evolution, Springer International Publishing, pp 14-26

Esslimani I, Brun A, Boyer A (2011) Densifying a behavioral recommender system by social networks link prediction methods. Social Network Analysis and Mining 1(3):159-172

Faloutsos M, Faloutsos P, Faloutsos C (1999) On power-law relationships of the internet topology. In: ACM SIGCOMM computer communication review, ACM, vol 29, pp 251-262

Feng X, Zhao J, Fang Z, Xu K (2014) Time-aware reciprocity prediction in trust network. In: Advances in Social Networks Analysis and Mining (ASONAM), pp 234-237

Gong NZ, Xu W (2014) Reciprocal versus parasocial relationships in online social networks. Social Network Analysis and Mining 4(1):1-14

Hasan MA, Zaki MJ (2011) Social Network Data Analytics, Springer US, chap A Survey of Link Prediction in Social Networks

Hasan MA, Chaoji V, Salem S, Zaki M (2006) Link prediction using supervised learning. In: In Proc. of SDM 06 workshop on Link Analysis, Counterterrorism and Security

Hopcroft J, Lou T, Tang J (2011) Who will follow you back?: Reciprocal relationship prediction. In: Proc. of CIKM, pp 1137-1146

Kaplan EL, Meier P (1958) Nonparametric estimation from incomplete observations. Journal of the American Statistical Association 53(282):457-481, URL http://www.jstor.org/stable/2281868

Kuhnt MR, Brust OA (2014) Low reciprocity rates in acquaintance networks of young adults: fact or artifact? Social Network Analysis and Mining 4(1):167

Leider S, Mobius MM, Rosenblat T, Do QA (2007) How much is a friend worth? directed altruism and enforced reciprocity in social networks. revision of NBER Working Paper 13135, Cambridge, Mass, National Bureau of Economics Research

Leskovec J, Kleinberg J, Faloutsos C (2007) Graph evolution: Densification and shrinking diameters. ACM Transactions on Knowledge Discovery from 
Data (TKDD)

Li M, Jia Y, Wang Y, Zhao Z, Cheng X (2016) Predicting links and their building time: A path-based approach. In: Proceedings of the Thirtieth AAAI Conference on Artificial Intelligence, AAAI Press, AAAI'16, pp 4228-4229

Liaghat Z, Rasekh AH, Mahdavi A (2013) Application of data mining methods for link prediction in social networks. Social Network Analysis and Mining $3(2): 143-150$

Liben-Nowell D, Kleinberg J (2003) The link prediction problem for social networks. In: Proc. of CIKM, pp 556-559

Nurcan Durak APCS Tamara G Kolda (2013) A scalable null model for directed graphs matching all degree distributions: In, out, and reciprocal. In: IEEE Workshop on Network Science, IEEE Press, Piscataway, NJ, pp 23-30

Pencina MJ, D'Agostino RB (2004) Overall-c as a measure of discrimination in survival analysis: model specific population value and confidence interval estimation. Statistics in Medicine 23(13):2109-2123

Roshanaei M, Mishra S (2015) Studying the attributes of users in twitter considering their emotional states. Social Network Analysis and Mining 5(1):34

Schwarz G (1978) Estimating the dimension of a model. The Annals of Statistics 6(2):461-464

Shahriari M, Sichani OA, Gharibshah J, Jalili M (2016) Sign prediction in social networks based on users reputation and optimism. Social Network Analysis and Mining 6(1):91

Song D, Meyer DA (2015) Link sign prediction and ranking in signed directed social networks. Social Network Analysis and Mining 5(1):52

Sun Y, Barber R, Gupta M, Aggarwal CC, Han J (2011) Co-author relationship prediction in heterogeneous bibliographic networks. In: International Conference on Advances in Social Networks Analysis and Mining, pp 121128

Sun Y, Han J, Aggarwal CC, Chawla NV (2012) When will it happen?: Relationship prediction in heterogeneous information networks. In: ACM International Conference on Web Search and Data Mining, WSDM, pp 663-672

Symeonidis P, Mantas N (2013) Spectral clustering for link prediction in social networks with positive and negative links. Social Network Analysis and Mining 3(4):1433-1447

Trivers RL (1971) The evolution of reciprocal altruism. Quarterly Review of Biology 46:33-57

Tu K, Ribeiro B, Jensen D, Towsley D, Liu B, Jiang H, Wang X (2014) Online dating recommendations: Matching markets and learning preferences. In: Proceedings of the 23rd International Conference on World Wide Web, ACM, New York, NY, USA, WWW '14 Companion, pp 787-792

Tuna T, Akbas E, Aksoy A, Canbaz MA, Karabiyik U, Gonen B, Aygun R (2016) User characterization for online social networks. Social Network Analysis and Mining 6(1):104

Valverde-Rebaza J, de Andrade Lopes A (2013) Exploiting behaviors of communities of twitter users for link prediction. Social Network Analysis and Mining 3(4):1063-1074 
Vinzamuri B, Reddy CK (2013) Cox regression with correlation based regularization for electronic health records. In: Intl. Conf. on Data Mining (ICDM), pp $757-766$

Wang P, Li Y, Reddy CK (2017a) Machine learning for survival analysis: A survey. ACM Computing Surveys

Wang Z, Wang C (2010) Buckley-james boosting for survival analysis with high-dimensional biomarker data. Statistical App in Genetics and Molecular Biology 9(1)

Wang Z, Chen C, Li W (2017b) Predictive network representation learning for link prediction. In: Proceedings of the 40th International ACM SIGIR Conference on Research and Development in Information Retrieval, SIGIR '17, pp 969-972

Xia P, Ribeiro B, Chen C, Liu B, Towsley D (2013) A study of user behavior on an online dating site. In: 2013 IEEE/ACM International Conference on Advances in Social Networks Analysis and Mining (ASONAM 2013), pp 243-247

Xia P, Liu B, Sun Y, Chen C (2015) Reciprocal recommendation system for online dating. In: IEEE/ACM International Conference on Advances in Social Networks Analysis and Mining, ACM, ASONAM '15, pp 234-241

Xia P, Zhai S, Liu B, Sun Y, Chen C (2016) Design of reciprocal recommendation systems for online dating. Social Network Analysis and Mining 6(1):32

Yang Y, Zou H (2013) A cocktail algorithm for solving the elastic net penalized cox regression in high dimensions. Stat and Its Interface 6(2):167-173

Zang X, Yamasaki T, Aizawa K, Nakamoto T, Kuwabara E, Egami S, Fuchida Y (2017) You will succeed or not? matching prediction in a marriage consulting service. In: 2017 IEEE Third International Conference on Multimedia Big Data (BigMM), pp 109-116

Zhang B, Choudhury S, Hasan MA, Ning X, Agarwal K, Purohit S, Cabrera PGP (2016) Trust from the past: Bayesian personalized ranking based link prediction in knowledge graphs. In: SDM Workshop on Mining Networks and Graphs (MNG 2016)

Zhao K, Wang X, Yu M, Gao B (2014) User recommendations in reciprocal and bipartite social networks-an online dating case study. IEEE Intelligent Systems 29(2):27-35

Zhu YX, Zhang XG, Sun GQ, Tang M, Zhou T, Zhang ZK (2014) Influence of reciprocal links in social networks. PloS one $9(7)$

Zlatić V, Štefančić H (2009) Influence of reciprocal edges on degree distribution and degree correlations. Physical Review E 80(1)

Zou H, Hastie T (2005) Regularization and variable selection via the elastic net. Journal of the Royal Statistical Society, Series B 67:301-320 